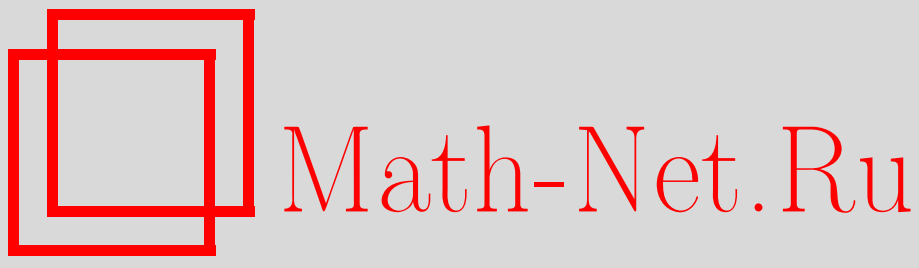

А. В. Горшков, Стабилизация полулинейного параболического уравнения, заданного во внешности ограниченной области, посредством управления с границы, $M a-$ тем. сб., 2003, том 194, номер 10, 49-76

DOI: https://doi.org/10.4213/sm773

Использование Общероссийского математического портала Math-Net.Ru подразумевает, что вы прочитали и согласны с пользовательским соглашением

http: //www.mathnet.ru/rus/agreement

Параметры загрузки:

IP : 3.85 .183 .62

26 апреля 2023 г., 13:42:14 


\title{
А.В. Горшков
}

\section{Стабилизация полулинейного параболического уравнения, заданного во внешности ограниченной области, посредством управления с границы}

\begin{abstract}
В работе исследуется задача стабилизации полулинейного уравнения, заданного во внешности ограниченной области. Невозможность экспоненциальной стабилизации вида $e^{-\sigma t}$ решения параболического уравнения, заданного в неограниченной области, ни при каком управлении на границе приводит к постановке задачи степенной стабилизации за счет управления на границе. Для заданного начального условия и параметра скорости стабилизации $k>0$ доказана возможность выбора такого граничного управления, что решение стремится к нулю со степенной скоростью $1 / t^{k}$.

Библиография: 27 названий.
\end{abstract}

\section{Введение}

В настояшей работе изучается задача стабилизации параболического уравнения во внешности ограниченной области. А именно пусть $B \subset \mathbb{R}^{d}$ - ограниченная односвязная область с гладкой границей класса $C^{\infty}$. В области $\Omega=\mathbb{R}^{d} \backslash B$ рассматривается полулинейное параболическое уравнение

$$
\partial_{t} y(t, x)-\Delta y(t, x)-|y|^{\gamma-1} y=0, \quad x \in \Omega, \quad t \in \mathbb{R}_{+},
$$

с начальным и граничньм условиями

$$
\begin{aligned}
\left.y\right|_{t=0} & =y_{0}(x), \\
\left.y\right|_{x \in \partial \Omega} & =u(t, x) .
\end{aligned}
$$

Здесь $u(t, x)$ является управлением.

Для заданного $k>0$ ишется такое управление $u(t, x)$, чтобы решение $y(t, x)$ полученной краевой задачи (1)-(3) удовлетворяло следующей оценке:

$$
\|y(t, \cdot)\| \leqslant \frac{C}{t^{k}} .
$$

В последние десятилетия активно исследовались задачи о точной нуль-управляемости и стабилизации в ограниченных областях. Постановка задачи и первые результаты, касающиеся точной управляемости (в частности, точной нуль-управляемости), были получены А. Г. Бутковским [1], Ю. В. Егоровым [2], [3], Л. И. Гальчуком [4]. 
Задача о точной управляемости заключается в поиске такого управления (управления с гранищы или управления с распределенньми параметрами), что за конечное время $T$ система переходит из начального состояния $y_{0}(x)$ в заданное финальное состояние $\widehat{y}(x)$ :

$$
\left.y\right|_{t=T}=\widehat{y}(x) .
$$

Если финальное состояние $\widehat{y}(x)$ тождественно равно нулю, то приведенная выше задача называется задачей о точной нуль-управляемости.

Исследования задач о точной управляемости для параболических уравнений были продолжены Д. Расселом, Г. Фатторини (см. [5], [6]). Для гиперболических систем результаты о точной управляемости получены в работах И. Лазиешки, Р. Триджиани [7], [8]. Во всех этих работах область, в которой рассматривались дифференциальные уравнения, была ограниченной. Задача о точной управляемости для абстрактных систем, заданных в бесконечномерных банаховых пространствах, изучалась Р. Триджиани [9]. Для более широких классов параболических уравнений и системы Навье-Стокса точная управляемость в ограниченных областях доказана в работах А. В. Фурсикова [10] и А. В. Фурсикова, О. Ю. Эмануилова (см. [11], [12] и приведенный там список литературы).

Исследование задачи о точной управляемости систем, описьваемых дифференциальньми уравнениями в частных производных, проходило параллельно с исследованием задачи о стабилизации в ограниченных областях. Последняя является родственной к задаче нуль-управляемости в том смысле, что также ищется управляющее воздействие, переводящее начальное состояние системы в нулевое финальное, но только не за конечное время $T$, а за бесконечное. При этом обьчно налагаются некоторые дополнительные условия типа робастности. Здесь следует отметить работы Л. Лазиешки [13], А. В. Фурсикова [14], [15]. Приводимый нами обзор не претендует на полноту охвата всех имеющихся на сегодняшний день работ по управляемости и стабилизации.

Более подробное рассмотрение полученных к середине 70-х годов результатов, связанных с этой областью, можно найти в работе Д. Рассела [16] и к концу 90-х - в работе А.В. Фурсикова и О. Ю. Эмануилова [12], а также в приведенном там списке литературы.

Для случая граничного управления интерес представляет не просто возможность стабилизации при $t \rightarrow \infty$, которая, например, для уравнения теплопроводности реализуется уже при граничном управлении равном нулю, а сама скорость этой стабилизации. В ограниченных областях таковой является экспоненциальная скорость вида

$$
\|y(t, \cdot)\| \leqslant e^{-\sigma t}, \quad \sigma>0
$$

как это показано в работах Л. Лазиешки [13] и А. В. Фурсикова [14], [15].

Однако случай неограниченной области имеет две сушественные особенности, сильно отличающие его от случая ограниченной области:

1. Отсутствие точной нуль-управляемости. Подтверждением этому служит тот факт, что уже для уравнения теплопроводности, заданного на полупространстве, выполнение условия (4) с нулевым финальным состоянием $\widehat{y} \equiv 0$ невозможно ни при каком управлении с границы (см., например, работу Г. Серегина, В. Сверака [17]). Этот факт мотивирует исследование задачи стабилизации вместо задачи 
о точной нуль-управляемости с границы, когда рассматриваемая область определения становится неограниченной.

2. Отсутствие экспоненциальной стабилизации. В $§ 1$ этой работы для уравнения теплопроводности, определенного на полуограниченном стержне, приведен пример начального условия, при котором экспоненциальная скорость стабилизации при $t \rightarrow \infty$ посредством управления с границы невозможна. Максимально возможной здесь является степенная скорость вида $1 / t^{k}$, где $k>0$. Причем скорость степенной стабилизации существенно зависит от выбираемого граничного управления в том смысле, что не существует единой управляющей функции $u(t)$ такой, что решение стабилизируется к нулю быстрее функции $1 / t^{k}$ с любым $k>0$.

Последний результат представлен в работе автора [18], в которой исследована задача стабилизации с границы одномерного уравнения теплопроводности, определенного на полуограниченном стержне $\mathbb{R}_{+}$. Для заданного параметра скорости стабилизации $k>0$ доказана возможность выбора такого граничного управления, что решение стабилизируется к нулю при $t \rightarrow \infty$ со степенной скоростью $1 / t^{k}$.

В настоящей работе получены результаты степенной стабилизации при $t \rightarrow \infty$ в многомерном случае для нелинейного параболического уравнения, определенного во внешности ограниченной области с управлением на ее границе. Используя методику, разработанную и примененную А.В. Фурсиковым при решении задачи стабилизации решений параболических уравнений и системы Навье-Стокса в ограниченной области (см. [14], [15]), мы сведем решение задачи стабилизации к решению задачи Коши со специальным начальным условием, принадлежащим устойчивому инвариантному многообразию $M_{-}$.

Основным преимуществом используемого метода решения задачи стабилизации является тот факт, что получаемое с его помощью управление является управлением с обратной связъю. Это дает возможность наряду с теоретическими результатами о сушествовании управления получать численные решения задачи. Подробно вопросы, связанные с управлением с обратной связью, рассмотрены в работах А. В. Фурсикова [15], [19], в которых исследуется задача стабилизации решений системы Навье-Стокса в ограниченных областях.

При решении задачи о стабилизации в настоящей работе использован ряд методов исследования задачи Коши для параболических уравнений и системы Навье-Стокса, которые представлены в следующих работах. В работах К. Уэйна [20] и Дж. Экманна, К. Уэйна [21] исследована задача Коши для нелинейного параболического уравнения и смежные с ней вопросы устойчивости и асимптотики поведения решения при $t \rightarrow \infty$. С помошью специального пространственно-временного преобразования нелинейное уравнение сводилось к уравнению, линейная часть которого обладала более удобной спектральной структурой. В соболевских пространствах с весом $H_{m}^{r}$, точное определение которых будет дано ниже, посредством такого преобразования отделялась дискретная часть спектра от непрерывной и строились инвариантные многообразия как линейной, так и нелинейной задач. В работе Т. Галлай, К. Уэйна [22] с помощью этого же преобразования исследована асимптотика поведения решения системы Навье-Стокса. Эта конструкция используется нами при решении задачи Коши, поэтому она будет подробно описана в нашей статье.

При исследовании задачи стабилизации мы ограничимся рассмотрением нели- 
нейности специального вида $|y|^{\gamma-1} y$ (условия на $\gamma$ будут приведены ниже). Это обусловлено тем, что упоминаемое выше преобразование сводит нашу автономную систему снова к автономной. Этот факт значительно упростит наше исследование и сделает вьвод результата о стабилизации более прозрачньм, потому что неавтономность системы необходимо влечет более сложную геометрическую структуру получаемых инвариантных многообразий.

Статья состоит из шести параграфов. В $\S 1$ для уравнения теплопроводности, заданного на полупрямой $\mathbb{R}_{+}$, приводится пример начального условия, при котором экспоненциальная стабилизация невозможна ни при каком граничном управлении. В последующих параграфах доказывается локальное существование степенной стабилизации для решения полулинейного параболического уравнения, определенного во внешности ограниченной области. А именно в $§ 2$ приводится постановка задачи степенной стабилизации и формулируется основной результат. В 33 исследуется линеаризованная задача Коши и спектр инфинитезимального оператора разрешающей полугрупшы. В $\S 4$ доказывается существование решения нелинейной задачи Коши и соответствуюшей разрешающей полугруппы. В $\S 5$ на основе спектральной теории, описанной в $\S 3$, строятся многообразия, инвариантные относительно полученной полугруппы, определенные в достаточно малой окрестности нуля. В заключительном $\S 6$ строится продолжение начального условия внутрь ограниченной области, обеспечивающее попадание полученной функции на инвариантное многообразие $M_{-}$.

Автор выражает благодарность А. В. Фурсикову за ценные замечания при подготовке статьи.

\section{§1. Пример отсутствия экспоненциальной стабилизации}

В этом параграфе мы приведем пример одной краевой задачи для уравнения теплопроводности, определенного на полупрямой, для которого экспоненциальная стабилизация невозможна ни при каком граничном управлении.

Рассмотрим уравнение теплопроводности

$$
\partial_{t} y(t, x)-\partial_{x x}^{2} y(t, x)=0, \quad(t, x) \in \mathbb{R}_{+} \times \mathbb{R}_{+}=Q
$$

с начальным условием

$$
\left.y\right|_{t=0}=y_{0}(x)
$$

и граничным условием

$$
\left.y\right|_{x=0}=u(t)
$$

где $u(t)$ является управлением.

Определим следующие соболевские пространства, в которых мы будем рассматривать уравнение теплопроводности:

$$
\begin{aligned}
H^{1,2}(Q)= & \left\{y(t, x):\|y\|_{H^{1,2}(Q)}^{2}=\|y\|_{L_{2}(Q)}^{2}+\left\|\partial_{t} y\right\|_{L_{2}(Q)}^{2}+\left\|\partial_{x} y\right\|_{L_{2}(Q)}^{2}\right. \\
& \left.+\left\|\partial_{x x} y\right\|_{L_{2}(Q)}^{2}<\infty\right\} \\
H^{1}\left(\mathbb{R}_{+}\right)= & \left\{y_{0}(x) \in L_{2}\left(\mathbb{R}_{+}\right):\left\|y_{0}\right\|_{H^{1}\left(\mathbb{R}_{+}\right)}^{2}=\left\|y_{0}\right\|_{L_{2}\left(\mathbb{R}_{+}\right)}^{2}+\left\|\partial_{x} y_{0}\right\|_{L_{2}\left(\mathbb{R}_{+}\right)}^{2}<\infty\right\}
\end{aligned}
$$


и $H^{3 / 4}\left(\mathbb{R}_{+}\right)$- пространство функций $u(t)$, квадратично суммируемых вместе с дробной производной порядка $3 / 4$. Выбор $H^{3 / 4}\left(\mathbb{R}_{+}\right)$в качестве пространства управлений обусловлен тем фактом, что оно является пространством следов для пространства $H^{1,2}(Q)$ (определения пространств Соболева с дробным показателем и свойства их следов на границе см. в [23], [24]).

ОПРЕДЕЛЕНИЕ 1.1. Мы будем говорить, что уравнение (5), дополненное начальным условием (6) из пространства $H^{1}\left(\mathbb{R}_{+}\right)$, не обладает свойством әкспоненциальной стабилизируемости в пространстве $H^{1,2}(Q)$, если ни при каком $\alpha>0$ не сушествует ни одной управляюшей функции $u(t) \in H^{3 / 4}\left(\mathbb{R}_{+}\right)$такой, чтобы решение $y(t, x) \in H^{1,2}(Q)$ краевой задачи $(5)-(7)$ при всех $x \in \mathbb{R}_{+}$удовлетворяло условию стабилизации:

$$
\int_{0}^{\infty}\left|e^{\alpha t} y(t, x)\right|^{2} d t<\infty .
$$

В этом условии под значением функции $y(t, x)$ при фиксированном $x$ понимается значение ее следа.

Положим начальное условие равньм следующей функции:

$$
y_{0}(x)= \begin{cases}(x-1)^{2}, & x \in(0,1), \\ 0, & x \geqslant 1 .\end{cases}
$$

ТЕОРема 1.1. Уравнение (5) с начальной функиией (9) не обладает свойством әкспоненциальной стабилизируемости в пространстве $H^{1,2}(Q)$.

ДокАЗАТЕЛЬСтво. Зададимся произвольным $\alpha>0$. Мы докажем, что ни при каком граничном условии $u(t)$ решение задачи $(5)-(7)$ с начальным условием (9) не удовлетворяет условию стабилизации (8) на множестве $x \in \Xi \subset \mathbb{R}_{+}$ненулевой меры.

Предположим противное. Рассмотрим преобразование Лапласа

$$
\widehat{y}(\tau, x)=\int_{0}^{\infty} e^{-\tau t} y(t, x) d t,
$$

сводящее задачу (5), (6) к обыкновенному дифференциальному уравнению с параметром $\tau$ :

$$
\partial_{x x}^{2} \widehat{y}(\tau, x)-\tau \widehat{y}(\tau, x)=-y_{0}(x) .
$$

Для функций $y(t, x)$ из пространства $H^{1,2}(Q)$ преобразование Лапласа $\widehat{y}(\tau, x)$ при почти всех $x$ является аналитической функцией в комплексной области $D=$ $\{\tau \in \mathbb{C}: \operatorname{Re} \tau>0\}$. Более того, в предположении (8) функция $\widehat{y}(\tau, x)$ при почти всех $x$ должна быть аналитична в большей области $\widetilde{D}=\{\tau \in \mathbb{C}: \operatorname{Re} \tau>-\alpha\}$.

Решение уравнения (11) дается явной формулой:

$$
\begin{aligned}
\widehat{y}(\tau, x)= & \widehat{u}(\tau) e^{-\sqrt{\tau} x}-\frac{e^{-\sqrt{\tau} x}}{2 \sqrt{\tau}} \int_{0}^{\infty} e^{-\sqrt{\tau} z} y_{0}(z) d z \\
& +\frac{e^{-\sqrt{\tau} x}}{2 \sqrt{\tau}} \int_{0}^{x} e^{\sqrt{\tau} z} y_{0}(z) d z+\frac{e^{\sqrt{\tau} x}}{2 \sqrt{\tau}} \int_{x}^{\infty} e^{-\sqrt{\tau} z} y_{0}(z) d z
\end{aligned}
$$


Здесь функция $\widehat{u}(\tau)$ - преобразование Лапласа управления $u(t)$. Функция $\sqrt{\tau}$ является ветвящейся с порядком ветвления равным двум. При совершении одного оборота вокруг нуля функция $\sqrt{\tau}$ переходит в функцию $-\sqrt{\tau}$. Поэтому все члены, входящие в правую часть формулы (12), также являются ветвящимися функциями с порядком ветвления равным двум. А в предположении экспоненциальной стабилизации функция $\widehat{y}$ должна быть аналитична в области $\widetilde{D}$ и, в частности, в окрестности нуля. Для этого необходимо, чтобы формула (12) была инвариантна относительно замены $\sqrt{\tau}$ на $-\sqrt{\tau}$. Иньми словами, должно выполняться равенстBO

$$
\begin{aligned}
& \widehat{u}(\tau) e^{-\sqrt{\tau} x}-\frac{e^{-\sqrt{\tau} x}}{2 \sqrt{\tau}} \int_{0}^{\infty} e^{-\sqrt{\tau} z} y_{0}(z) d z+\frac{e^{-\sqrt{\tau} x}}{2 \sqrt{\tau}} \int_{0}^{x} e^{\sqrt{\tau} z} y_{0}(z) d z \\
& +\frac{e^{\sqrt{\tau} x}}{2 \sqrt{\tau}} \int_{x}^{\infty} e^{-\sqrt{\tau} z} y_{0}(z) d z=\widehat{u}(\tau) e^{\sqrt{\tau} x}+\frac{e^{\sqrt{\tau} x}}{2 \sqrt{\tau}} \int_{0}^{\infty} e^{\sqrt{\tau} z} y_{0}(z) d z \\
& -\frac{e^{\sqrt{\tau} x}}{2 \sqrt{\tau}} \int_{0}^{x} e^{-\sqrt{\tau} z} y_{0}(z) d z-\frac{e^{-\sqrt{\tau} x}}{2 \sqrt{\tau}} \int_{x}^{\infty} e^{\sqrt{\tau} z} y_{0}(z) d z
\end{aligned}
$$

Отсюда находим функцию $\widehat{u}(\tau)$ :

$$
\widehat{u}(\tau)=\int_{0}^{\infty} \frac{e^{-\sqrt{\tau} z}-e^{\sqrt{\tau} z}}{2 \sqrt{\tau}} y_{0}(z) d z
$$

Подставляя полученное выражение для функции $\widehat{u}(\tau)$ в формулу $(12)$, получаем следуюшее представление для $\widehat{y}(\tau, x)$ :

$$
\widehat{y}(\tau, x)=\int_{x}^{\infty} \frac{e^{\sqrt{\tau}(x-z)}-e^{-\sqrt{\tau}(x-z)}}{2 \sqrt{\tau}} y_{0}(z) d z
$$

Подставляя (9) в (13) и производя элементарные вычисления, получаем следуюшее представление для функции $\widehat{y}(\tau, x)$ :

$$
\widehat{y}(\tau, x)= \begin{cases}\frac{1}{\tau}(x-1)^{2}+\frac{2}{\tau^{2}}-\frac{1}{\tau^{2}} e^{-\sqrt{\tau}(1-x)}-\frac{1}{\tau^{2}} e^{\sqrt{\tau}(1-x)}, & x \in(0,1) \\ 0, & x \geqslant 1 .\end{cases}
$$

Как нетрудно видеть, функция $\widehat{y}(i \xi, x)$, определяемая формулой $(14)$, является экспоненциально растущей при $\xi \rightarrow \infty, x \in(0,1)$. С другой стороны, из самого определения преобразования Лапласа (10) следует, что функция $\widehat{y}(i \xi, x)$ является преобразованием Фурье функции $y(t, x)$, принадлежащей пространству $H^{1,2}(Q)$. Поэтому $\widehat{y}(i \xi, x)$ должна быть квадратично интегрируема по $\xi$ при почти всех $x$. Полученное противоречие доказывает теорему.

Невозможность экспоненциальной стабилизации приводит нас к постановке задачи степенной стабилизации. 


\section{§2. Постановка задачи степенной стабилизации}

Пусть $B \subset \mathbb{R}^{d}$ - ограниченная односвязная область с гладкой границей класса $C^{\infty}$. В области $\Omega=\mathbb{R}^{d} \backslash B$ рассматривается полулинейное параболическое уравнение вида

$$
\partial_{t} y(t, x)-\Delta y(t, x)-|y|^{\gamma-1} y=0, \quad x \in \Omega, \quad t \in \mathbb{R}_{+},
$$

с начальным и граничньм условиями

$$
\begin{aligned}
\left.y\right|_{t=0} & =y_{0}(x), \\
\left.y\right|_{x \in \partial \Omega} & =u(t, x) .
\end{aligned}
$$

Здесь $u(t, x)$ является управлением. ${ }^{1}$

На $\gamma$ будем налагать следующее ограничение:

$$
\gamma>\max \left(1+\frac{2}{d}, 2+\frac{d}{2}\right)
$$

Нашу задачу мы будем рассматривать в следующих соболевских пространстBax:

$$
\begin{aligned}
H^{r}(\Omega) & =\left\{f(x):\|f(x)\|_{H^{r}(\Omega)}^{2}=\sum_{|\alpha| \leqslant r}\left\|\partial^{\alpha} f(x)\right\|_{L_{2}(\Omega)}^{2}<\infty\right\}, \\
H_{m}^{r}(\Omega) & =\left\{f(x):\|f(x)\|_{H_{m}^{r}(\Omega)}^{2}=\sum_{|\alpha| \leqslant r}\left\|\left(1+|x|^{2}\right)^{m / 2} \partial^{\alpha} f(x)\right\|_{L_{2}(\Omega)}^{2}<\infty\right\}, \\
H^{r-1 / 2}(\partial \Omega) & =\left\{\gamma_{\partial \Omega} f(x): f \in H^{r}(\Omega)\right\},
\end{aligned}
$$

где $m \geqslant 0, r \geqslant 0$ - некоторые целые числа, а $\gamma \partial \Omega$ обозначает оператор сужения функций из пространства $H^{r}(\Omega)$ на границу $\partial \Omega$ (более подробно определения соболевских пространств и операторы сужения рассмотрены в книгах Ж.-Л. Лионса, Э. Мадженеса [23] и А. В. Фурсикова [24]). Здесь и далее символ $\alpha=\left(\alpha_{1}, \ldots, \alpha_{d}\right)$ обозначает мультииндекс, $|\alpha|=\sum_{i=1}^{d} \alpha_{i}, \partial^{\alpha}=\frac{\partial^{|\alpha|}}{\partial_{x_{1}}^{\alpha_{1}} \cdots \partial_{x_{d}}^{\alpha_{d}}}-$ оператор дифференцирования. Аналогичньм образом определяются пространства $H^{r}\left(\mathbb{R}^{d}\right), H_{m}^{r}\left(\mathbb{R}^{d}\right)$, которые будут использоваться наряду с введенными $H^{r}(\Omega), H_{m}^{r}(\Omega)$. В тех случаях, когда из контекста изложения будет понятна рассматриваемая область определения, мы будем пользоваться сокрашенными обозначениями $H^{r}, H_{m}^{r}$.

Далее, обозначим

$$
\begin{gathered}
L_{2}\left(0, \infty ; H^{r}(\Omega)\right)=\left\{y(t, x):\|y\|_{L_{2}\left(0 ; \infty ; H^{r}(\Omega)\right)}^{2}\right. \\
\left.=\int_{0}^{\infty}\|y(t, \cdot)\|_{H^{r}(\Omega)}^{2} d t<\infty\right\}, \\
L_{2}\left(0, \infty ; H^{r-1 / 2}(\partial \Omega)\right)=\left\{u(t, x):\|u\|_{L_{2}\left(0 ; \infty ; H^{r-1 / 2}(\partial \Omega)\right)}^{2}\right. \\
\left.=\int_{0}^{\infty}\|u(t, \cdot)\|_{H^{r-1 / 2}(\partial \Omega)}^{2} d t<\infty\right\} .
\end{gathered}
$$

\footnotetext{
${ }^{1}$ Отметим, что все получаемые в работе резултаты являются локальными, и поэтому выбор знака, стоящего перед нелинейньм членом $|y|^{\gamma-1} y$, не является существенньм.
} 
Также в работе будут использоваться пространство $C\left([0, \infty) ; H^{r}(\Omega)\right)$ как пространство непрерывных отображений $y(t, \cdot)$ из $[0, \infty)$ в $H^{r}(\Omega)$ и пространство $C\left([0, \infty) ; H^{r-1 / 2}(\partial \Omega)\right)$ непрерьвных отображений $u(t, \cdot)$ из $[0, \infty)$ в $H^{r-1 / 2}(\partial \Omega)$. Аналогичным образом определяются $L_{2}\left(0, \infty ; H_{m}^{r}(\Omega)\right)$ и $C\left([0, \infty) ; H_{m}^{r}(\Omega)\right)$.

Мы будем решать следуюшую задачу стабилизации. Пусть заданы параметр скорости стабилизации $k>0$ и начальное условие $y_{0}(x) \in H_{m}^{r}(\Omega)$. Нужно найти управление $u\left(t, x^{\prime}\right), x^{\prime} \in \partial \Omega, t>0$, такое, что решение $y(t, x)$ уравнения (15) с начальным и граничным условиями (16), (17) при достаточно больших $t>0$ удовлетворяло бы условию степенной стабилизации:

$$
\|y(t, \cdot)\|_{H^{r}(\Omega)} \leqslant \frac{C}{t^{k}}\left\|y_{0}(\cdot)\right\|_{H_{m}^{r}(\Omega)}
$$

Основным результатом работы является

Теорема 2.1. Для любых $k>0$, иельх $r, d / 2<r<\gamma, m>m_{0}$, әде $m_{0}=m_{0}(k)$ достаточно велико, и произвольного начального условия $y_{0}(x) \in$ $H_{m}^{r}(\Omega)$ с достаточно малой нормой $\left\|y_{0}(\cdot)\right\|_{H_{m}^{r}(\Omega)}$ существует решение

$$
\begin{aligned}
y(t, x) & \in L_{2}\left(0, \infty ; H^{r}(\Omega)\right) \cap C\left([0, \infty) ; H^{r}(\Omega)\right), \\
u\left(t, x^{\prime}\right) & \in L_{2}\left(0, \infty ; H^{r-1 / 2}(\partial \Omega)\right) \cap C\left([0, \infty) ; H^{r-1 / 2}(\partial \Omega)\right)
\end{aligned}
$$

задачи стабилизачии (15)-(17), (19).

При доказательстве теоремы 2.1 мы будем использовать метод, разработанный А.В. Фурсиковьм при исследовании задач стабилизации параболических уравнений и системы Навье-Стокса [14], [15], [19]. Он основан на специальном продолжении начального условия $y_{0}(x)$ внутрь области $B$, которое мы обозначим через $z_{0}(x)$. Способ построения такого продолжения будет описан ниже.

Далее рассмотрим задачу Коши

$$
\partial_{t} z(t, x)-\Delta z(t, x)-|z|^{\gamma-1} z=0, \quad x \in \mathbb{R}^{d}, \quad t \in \mathbb{R}_{+},
$$

с этим начальньп условием $z_{0}(x)$ :

$$
\left.z\right|_{t=0}=z_{0}(x), \quad x \in \mathbb{R}^{d}
$$

Мы будем искать такое продолжение $z_{0}(x)$, чтобы решение $z(t, x)$ задачи Коши (20), (21) стремилось к нулю с заданной скоростью $1 / t^{k}$. Далее, ограничив полученное решение задачи Коши на область $\Omega$, мы получим искомое решение задачи стабилизации $y(t, x)$ с управлением $u\left(t, x^{\prime}\right)=\left.z\left(t, x^{\prime}\right)\right|_{x^{\prime} \in \partial \Omega}$.

Основным средством исследования задачи Коши $(20),(21)$ будет замена переменных, использованная в работах К. Уэйна и Дж. Экманна [20], [21] для получения асимптотик поведения решения параболического уравнения при $t \rightarrow \infty$ :

$$
\begin{aligned}
\xi & =\frac{x}{\sqrt{t+1}}, \\
\tau & =\ln (t+1) .
\end{aligned}
$$


С введением новой функции $\widehat{z}(\tau, \xi)$, определяемой формулой

$$
z(t, x)=\frac{1}{(t+1)^{\frac{1}{\gamma-1}}} \widehat{z}\left(\ln (t+1), \frac{x}{\sqrt{t+1}}\right)
$$

замена (22) сводит уравнение (20) к уравнению

$$
\frac{\partial \widehat{z}(\tau, \xi)}{\partial \tau}=\Delta \widehat{z}+\frac{1}{2}(\xi, \nabla) \widehat{z}+\frac{1}{\gamma-1} \widehat{z}+|\widehat{z}|^{\gamma-1} \widehat{z}
$$

с тем же начальным условием

$$
\left.\widehat{z}\right|_{\tau=0}=z_{0}(\xi), \quad \xi \in \mathbb{R}^{d} .
$$

Обозначим линейньй оператор, стоящий в правой части $(23)$, через $\mathscr{L}$ :

$$
\mathscr{L}=\Delta+\frac{1}{2}(\xi, \nabla)+\frac{1}{\gamma-1} .
$$

Дальнейшее исследование будет опираться на свойства оператора $\mathscr{L}$ и порождаемой им полугруппы $e^{\tau \mathscr{L}}$.

В $\S 3$ рассматривается линеаризованная задача Коши и спектр оператора $\mathscr{L}$. Локальная теорема существования и единственности нелинейной задачи Коши (23), (24) и основные свойства разрешающей полугруппы $S^{\tau}$ изучены в $\oint 4$. В $\S 5$ устанавливается локальное сушествование инвариантного относительно действия полугрупшы $S^{\tau}$ многообразия $M_{-}$, обладаюшего свойством “экспоненциального стягивания к нулю" в $\tau$-представлении, что соответствует степенной сходимости в $t$-представлении. Доказательство основной теоремы 2.1 завершается построением в $\S 6$ продолжения $z_{0}(x)$ начального условия $y_{0}(x)$ таким образом, чтобы оно принадлежало инвариантному многообразию $M_{-}$.

\section{§3. Линейная задача Коши. Спектр оператора $\mathscr{L}$}

Рассмотрим задачу Коши:

$$
\begin{aligned}
\frac{\partial \widehat{z}}{\partial \tau} & =\mathscr{L} \widehat{z}, \\
\left.\widehat{z}\right|_{t=0} & =z_{0}(\xi) .
\end{aligned}
$$

Справедлива следующая

Теорема 3.1 (о спектре оператора $\mathscr{L}$ ). Для заданных иелых неотрииательньи $m, r$ спектр оператора $\mathscr{L}$, определенного на пространстве $H_{m}^{r}\left(\mathbb{R}^{d}\right)$, задается соотношением

$$
\sigma(\mathscr{L})=\left\{\lambda \in \mathbb{C}: \operatorname{Re} \lambda \leqslant-\frac{d}{4}-\frac{m}{2}+\frac{1}{\gamma-1}\right\} \cup\left\{-\frac{l}{2}-\frac{d}{2}+\frac{1}{\gamma-1}: l \in \mathbb{N} \cup\{0\}\right\} .
$$

Для иельх неотрицательных $l<m-d / 2$ значение

$$
\lambda_{l}=-\frac{l}{2}-\frac{d}{2}+\frac{1}{\gamma-1}
$$

является изолированным собственным значением кратности $C_{d+l-1}^{l}$. 


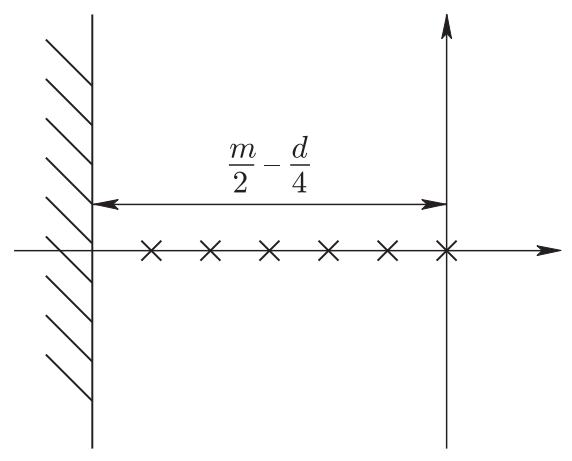

Рис. 1

Для доказательства теоремы вместо оператора $\mathscr{L}$ рассмотрим оператор $\Lambda$, являющийся сдвигом оператора $\mathscr{L}$ на величину $d / 2-1 /(\gamma-1)$ :

$$
\Lambda=\Delta+\frac{1}{2}(\xi, \nabla)+\frac{d}{2} .
$$

Теорема 3.1 вытекает из следующей теоремы о спектре оператора $\Lambda$ :

ТЕОРема 3.2. Для заданных иелых неотрицательных $m, r$ спектр оператора $\Lambda$, заданного на пространстве $H_{m}^{r}\left(\mathbb{R}^{d}\right)$, определяется соотношением

$$
\sigma(\Lambda)=\left\{\lambda \in \mathbb{C}: \operatorname{Re} \lambda \leqslant \frac{d}{4}-\frac{m}{2}\right\} \cup\left\{-\frac{l}{2}: l \in \mathbb{N} \cup\{0\}\right\} .
$$

Для иельх неотрицательных $l<m-d / 2$ значение $\lambda_{l}=-l / 2$ является изолированным собственным значением кратности $C_{d+l-1}^{l}$ (см. рис. 1).

Обозначим через $F$ преобразование Фурье функции $f \in H_{m}^{r}$ :

$$
(F f)(p)=\int_{\mathbb{R}^{d}} f(\xi) e^{-i p \xi} d \xi
$$

При этом преобразовании оператор $\Lambda$ перейдет в оператор $F \Lambda$ :

$$
(F \Lambda f)(p)=-\left(|p|^{2}+\frac{1}{2} p \cdot \nabla_{p}\right)(F f)(p) .
$$

Отметим, что пространство $H_{m}^{r}$ при действии преобразования $F$ переходит в пространство $H_{r}^{m}$. Действительно, при преобразовании Фурье дифференцирование порядка $r$ переходит в умножение на моном соответствуюшей степени, а весовая функция порядка $m$, наоборот, переходит в соответствуюший дифференциальный оператор. Тем самьм, параметр $r$ гладкости функций, входящих в $H_{m}^{r}$, выступает как параметр веса, а уже $m$ определяет гладкость функций.

Введем функции Эрмита

$$
\psi_{\alpha}(\xi)=\left(\partial_{\xi}^{\alpha} \psi_{0}\right)(\xi), \quad \psi_{0}(\xi)=\frac{1}{(4 \pi)^{d / 2}} e^{-|\xi|^{2} / 4} .
$$

Преобразование Фурье функций Эрмита определяется формулой

$$
\left(F \psi_{\alpha}\right)(p)=(i p)^{\alpha} e^{-|p|^{2}} .
$$


Лемма 3.1. Для произвольного иелого неотрицательного l функиии Эрмита $\psi_{\alpha}(\xi)$ при $|\alpha|=l$ являются собственными функииями оператора $\Lambda$.

ДокАЗАТЕЛЬСтво. С помощью прямых вычислений покажем, что функции $\left(F \psi_{\alpha}\right)(p)$ являются собственными функциями оператора $F \Lambda$ :

$$
\begin{aligned}
(F \Lambda)\left(F \psi_{\alpha}\right)(p)=-\left(|p|^{2}+\frac{1}{2} p \cdot \nabla_{p}\right)(i p)^{\alpha} e^{-|p|^{2}} & \\
= & -|p|^{2}(i p)^{\alpha} e^{-|p|^{2}}-\frac{1}{2} \sum_{k=1}^{d} p_{k} \frac{\partial}{\partial_{p_{k}}}(i p)^{\alpha} e^{-|p|^{2}} \\
= & -|p|^{2}(i p)^{\alpha} e^{-|p|^{2}}-\frac{1}{2} \sum_{k=1}^{d} p_{k} \prod_{j=1, j \neq k}^{d}\left(i p_{j}\right)^{\alpha_{j}} i \alpha_{k}\left(i p_{k}\right)^{\alpha_{k}-1} e^{-|p|^{2}} \\
& -\frac{1}{2} \sum_{k=1}^{d} p_{k}(i p)^{\alpha}\left(-2 p_{k}\right) e^{-|p|^{2}} \\
= & -|p|^{2}(i p)^{\alpha} e^{-|p|^{2}}-\frac{1}{2}(i p)^{\alpha} e^{-|p|^{2}} \sum_{k=1}^{d} \alpha_{k}+|p|^{2}(i p)^{\alpha} e^{-|p|^{2}} \\
= & -\frac{|\alpha|}{2}\left(F \psi_{\alpha}\right)(p) .
\end{aligned}
$$

Введем функции

$$
\psi_{-2 \lambda}=|p|^{-2 \lambda} e^{-|p|^{2}}
$$

Лемма 3.2. Пусть $\operatorname{Re} \lambda<d / 4-m / 2$. Тогда $\psi_{-2 \lambda}$ являются собственными функииями оператора $F \Lambda$.

ДокАЗАтЕльство. Аналогично выкладкам, проводимым при доказательстве леммы 3.1, можно показать, что вьполняется равенство $(F \Lambda) \psi_{-2 \lambda}=\lambda \psi_{-2 \lambda}$. Для завершения доказательства леммы необходимо доказать принадлежность функций $\psi_{-2 \lambda}$ пространству $H_{r}^{m}$ при $\operatorname{Re} \lambda<d / 4-m / 2$. Функции $\psi_{-2 \lambda}$ являются экспоненциально убывающими на бесконечности функциями с единственной особенностью в нуле. Поэтому принадлежность $\psi_{-2 \lambda}$ пространству $H_{r}^{m}$ эквивалентна квадратичной интегрируемости особенности в нуле $m$-й производной функций $\psi_{-2 \lambda}$ :

$$
\psi_{-2 \lambda} \in H_{r}^{m} \Leftrightarrow|p|^{-2 \lambda-m} \in L_{2}^{\operatorname{loc}}\left(\mathbb{R}^{d}\right) \Leftrightarrow \operatorname{Re} \lambda<\frac{d}{4}-\frac{m}{2} .
$$

Ввиду замкнутости спектра отсюда следует включение полуплоскости $\{\lambda$ : $\operatorname{Re} \lambda \leqslant d / 4-m / 2\}$ в непрерывную часть спектра $\Lambda$.

Возьмем $n \in \mathbb{Z}, 0 \leqslant n<m$, и определим операторы проектирования $P_{-}^{n}, P_{+}^{n}$ в пространстве $H_{m}^{r}\left(\mathbb{R}^{d}\right)$ следующим образом:

$$
\begin{aligned}
& P_{+}^{n}(f)=\sum_{|\alpha| \leqslant n}\left(\int_{\mathbb{R}^{d}} P_{\alpha}\left(\xi^{\prime}\right) f\left(\xi^{\prime}\right) d \xi^{\prime}\right) \psi_{\alpha}(\xi) \\
& P_{-}^{n}(f)=\left(I-P_{+}^{n}\right)(f) .
\end{aligned}
$$


Здесь $P_{\alpha}$ обозначают полиномы Эрмита степени $|\alpha|$ :

$$
P_{\alpha}(\xi)=\frac{1}{(4 \pi)^{d / 2}} e^{|\xi|^{2} / 4} \partial_{\xi}^{\alpha} e^{-|\xi|^{2} / 4}=e^{|\xi|^{2} / 4} \psi_{\alpha}(\xi) .
$$

Нетрудно видеть, что

$$
P_{+}^{n} f=0 \quad \Leftrightarrow \quad \int_{\mathbb{R}^{d}} f(\xi) \xi^{\alpha} d \xi=0, \quad|\alpha| \leqslant n .
$$

Действительно, ввиду линейной независимости функций Эрмита $\psi_{\alpha}(\xi)$ условие $P_{+}^{n} f=0$ эквивалентно ортогональности функции $f(\xi)$ всем полиномам Эрмита $P_{\alpha}(\xi)$ степени $|\alpha| \leqslant n$, что, в свою очередь, влечет

$$
\int_{\mathbb{R}^{d}} f(\xi) \xi^{\alpha} d \xi=0, \quad|\alpha| \leqslant n
$$

Для целых отрицательных $n$ положим $P_{+}^{n}=0, P_{-}^{n}=I$.

Лемма 3.3. Спектр оператора $\Lambda$ представим в виде обғединения спектров операторов $\Lambda P_{+}^{n} u \Lambda P_{-}^{n}$ :

$$
\sigma(\Lambda)=\sigma\left(\Lambda P_{+}^{n}\right) \cup \sigma\left(\Lambda P_{-}^{n}\right)
$$

ДокАЗАТЕЛЬСтво. Достаточно установить включения

$$
\Lambda P_{+}^{n} H_{m}^{r} \subset P_{+}^{n} H_{m}^{r}, \quad \Lambda P_{-}^{n} H_{m}^{r} \subset P_{-}^{n} H_{m}^{r} .
$$

Функции Эрмита $\psi_{\alpha}(\xi)$, на которые проецирует оператор $P_{+}^{n}$, являются собственными функциями оператора $\Lambda$. Отсюда следует справедливость первого включения в (27).

Покажем, что если для функции $f$ выполняются условия ортогональности (26), то те же условия выполняются и для функции $\Lambda f$. Обозначим через $\Lambda^{*}$ оператор, сопряженный $\Lambda$. Очевидно, $\Lambda^{*} \xi^{\alpha}$ является многочленом степени $|\alpha|$. Поэтому выполнено

$$
\int_{\mathbb{R}^{d}} \Lambda f(\xi) \xi^{\alpha} d \xi=\int_{\mathbb{R}^{d}} f(\xi) \Lambda^{*} \xi^{\alpha} d \xi=0, \quad|\alpha| \leqslant n .
$$

Отсюда следует последнее включение в (27).

Леммы 3.1, 3.2 устанавливают включение

$$
\sigma(\Lambda) \supset\left\{\lambda \in \mathbb{C}: \operatorname{Re} \lambda \leqslant \frac{d}{4}-\frac{m}{2}\right\} \cup\left\{-\frac{l}{2}: l \in \mathbb{N} \cup\{0\}\right\} .
$$

Возьмем $n \in \mathbb{Z}$, удовлетворяющее условию

$$
n+\frac{d}{2}<m \leqslant n+1+\frac{d}{2} .
$$


Спектр оператора $\Lambda P_{+}^{n}$ состоит из конечного числа собственных значений оператора $\Lambda$ :

$$
\sigma\left(\Lambda P_{+}^{n}\right)=\left\{0,-\frac{1}{2}, \ldots,-\frac{n}{2}\right\}
$$

Для спектра оператора $\Lambda P_{-}^{n}$ из предыдушей леммы с учетом (28) следует включение

$$
\sigma\left(\Lambda P_{-}^{n}\right) \supset\left\{\lambda \in \mathbb{C}: \operatorname{Re} \lambda \leqslant \frac{d}{4}-\frac{m}{2}\right\} .
$$

Пусть $e^{\tau \mathscr{L}}: H_{m}^{r}\left(\mathbb{R}^{d}\right) \rightarrow H_{m}^{r}\left(\mathbb{R}^{d}\right)$ - разрешаюшая полугруппа задачи Коши (25), ставящая в соответствие функции $z_{0}(\xi) \in H_{m}^{r}\left(\mathbb{R}^{d}\right)$ решение задачи Коши $(25)$ в момент времени $\tau$.

Полугруппа $e^{\tau \mathscr{L}}$ дается следующей формулой, которая выводится из известной формулы Пуассона для уравнения теплопроводности после замены (22):

$$
e^{\tau \mathscr{L}} f(\xi)=\frac{e^{\frac{\tau}{\gamma-1}}}{(4 \pi a(\tau))^{\frac{d}{2}}} \int_{\mathbb{R}^{d}} e^{-\frac{\left|\xi-\xi^{\prime}\right|^{2}}{4 a(\tau)}} f\left(\xi^{\prime} e^{\frac{\tau}{2}}\right) d \xi^{\prime},
$$

где $a(\tau)=1-e^{-\tau}$.

ЛЕмма 3.4 (об оценке полугруппы $e^{\tau \Lambda}$ в пространстве $H_{m}^{0}\left(\mathbb{R}^{d}\right)$ ).

1. Для произвольных целых $m \geqslant 0$ u $n \in \mathbb{N} \cup\{0\} \cup\{-1\}$, связанных соотночением $n+d / 2<m \leqslant n+1+d / 2, \alpha \in \mathbb{N}^{d}$ u $\varepsilon>0$ справедлива оченка ( некоторой константой $C$, не зависящей от $f$ ):

$$
\left\|\partial^{\alpha} e^{\tau \Lambda} P_{-}^{n} f\right\|_{H_{m}^{0}} \leqslant \frac{C}{a(\tau)^{\frac{|\alpha|}{2}}} e^{\frac{\tau}{2}\left(\frac{d}{2}-m+\varepsilon\right)}\|f\|_{H_{m}^{0}}
$$

2. Для произвольных цельхх $n \in \mathbb{N} \cup\{0\} \cup\{-1\}, m>n+1+d / 2, \alpha \in \mathbb{N}^{d}$ виьполнена следующая оценка ( с некоторой константой $C$, не зависящей от $f$ ):

$$
\left\|\partial^{\alpha} e^{\tau \Lambda} P_{-}^{n} f\right\|_{H_{m}^{0}} \leqslant \frac{C}{a(\tau)^{\frac{|\alpha|}{2}}} e^{-\frac{n+1}{2} \tau}\|f\|_{H_{m}^{0}} .
$$

Доказательство см. в [22].

СлЕДСТвИЕ 3.1 (об оценке полугрупшы $e^{\tau \Lambda}$ в пространстве $H_{m}^{r}\left(\mathbb{R}^{d}\right)$ ).

1. Для произвольных иелых $m \geqslant 0$ и $n \in \mathbb{N} \cup\{0\} \cup\{-1\}$, связанных соотношением $n+d / 2<m \leqslant n+1+d / 2, \alpha \in \mathbb{N}^{d}, r \in \mathbb{N} \cup\{0\}$ и $\varepsilon>0$ справедлива оценка (с некоторой константой $C$, не зависящей от $f$ ):

$$
\left\|e^{\tau \Lambda} P_{-}^{n} f\right\|_{H_{m}^{r}} \leqslant C e^{\frac{\tau}{2}\left(\frac{d}{2}-m+\varepsilon\right)}\|f\|_{H_{m}^{r}}
$$

2. Для произвольных цельхх $n \in \mathbb{N} \cup\{0\} \cup\{-1\}, m>n+1+d / 2, r \in \mathbb{N} \cup\{0\}$ выполнена следующая оченка ( $с$ некоторой константой $C$, не зависящей от $f$ ):

$$
\left\|e^{\tau \Lambda} P_{-}^{n} f\right\|_{H_{m}^{r}} \leqslant C e^{-\frac{n+1}{2} \tau}\|f\|_{H_{m}^{r}} .
$$


ДокаЗАТЕльство. Пусть $\tau \in[0,1]$. Так как для функции $P_{-}^{n} f$ выполнены условия ортогональности (26), то они же выполняются и для функции $\partial_{\xi}^{\beta}\left(P_{-}^{n} f\right)$. Отсюда следует включение $\partial_{\xi}^{\beta}\left(P_{-}^{n} f\right) \in P_{-}^{n} H_{m}^{r}$.

Домножим на $e^{\left(\frac{d}{2}-\frac{1}{\gamma-1}\right) \tau}$ и продифференцируем по $\xi$ обе части (31). После интегрирования по частям, положив в $(32) \alpha=0$, имеем:

$$
\begin{aligned}
&\left\|\partial_{\xi}^{\beta} e^{\tau \Lambda} P_{-}^{n} f(\xi)\right\|_{H_{m}^{0}}=\left\|\frac{e^{\frac{d \tau}{2}}}{(4 \pi a(\tau))^{\frac{d}{2}}} \int_{\mathbb{R}^{d}} e^{-\frac{\left|\xi-\xi^{\prime}\right|^{2}}{4 a(\tau)}} \partial_{\xi^{\prime}}^{\beta}\left(P_{-}^{n} f\left(\xi^{\prime} e^{\frac{\tau}{2}}\right)\right) d \xi^{\prime}\right\|_{H_{m}^{0}} \\
&=\left\|\frac{e^{\frac{d \tau}{2}}}{(4 \pi a(\tau))^{\frac{d}{2}}} \int_{\mathbb{R}^{d}} e^{-\frac{\left|\xi-\xi^{\prime}\right|^{2}}{4 a(\tau)}} e^{|\beta| \frac{\tau}{2}} \partial_{\xi^{\prime}}^{\beta}\left(P_{-}^{n} f\right)\left(\xi^{\prime} e^{\frac{\tau}{2}}\right) d \xi^{\prime}\right\|_{H_{m}^{0}} \\
&=\left\|e^{\tau\left(\Lambda+\frac{|\beta|}{2}\right)} \partial_{\xi}^{\beta}\left(P_{-}^{n} f\right)\right\|_{H_{m}^{0}} \leqslant e^{\frac{r}{2}}\left\|e^{\tau \Lambda} P_{-}^{n} \partial_{\xi}^{\beta}\left(P_{-}^{n} f\right)\right\|_{H_{m}^{0}} \\
& \leqslant C e^{\frac{r}{2}} e^{\frac{\tau}{2}\left(\frac{d}{2}-m+\varepsilon\right)}\left\|P_{-}^{n} f\right\|_{H_{m}^{r}} \leqslant C e^{\frac{r}{2}} e^{\frac{\tau}{2}\left(\frac{d}{2}-m+\varepsilon\right)}\|f\|_{H_{m}^{r}} \\
&|\beta| \leqslant r .
\end{aligned}
$$

Для $\tau>1$ из (32) следует оценка

$$
\begin{gathered}
\left\|\partial_{\xi}^{\beta} e^{\tau \Lambda} P_{-}^{n} f\right\|_{H_{m}^{0}} \leqslant \frac{C}{a(\tau)^{\frac{|\beta|}{2}}} e^{\frac{\tau}{2}\left(\frac{d}{2}-m+\varepsilon\right)}\|f\|_{H_{m}^{0}} \leqslant \frac{C}{\left(1-e^{-1}\right)^{\frac{r}{2}}} e^{\frac{\tau}{2}\left(\frac{d}{2}-m+\varepsilon\right)}\|f\|_{H_{m}^{r}}, \\
|\beta| \leqslant r .
\end{gathered}
$$

Из этих оценок следует оценка $(34)$ с константой $\max \left(C e^{\frac{r}{2}}, C\left(1-e^{-1}\right)^{-\frac{r}{2}}\right)$.

Оценка (35) выводится аналогичным образом из соответствующей оценки (33).

СлеДСТвиЕ 3.2 (об оценке полугруппы $e^{\tau \mathscr{L}}$ ). Для произвольных $s>0, r \in$ $\mathbb{N} \cup\{0\}$ существуют достаточно больиие натуральные $n=n(s)$ и $m=m(s)$ такие, что выполнена следующая оценка ( $с$ некоторой константой $C$, не зависящей от $f$ ):

$$
\left\|e^{\tau \mathscr{L}} P_{-}^{n} f\right\|_{H_{m}^{r}} \leqslant C e^{-s \tau}\|f\|_{H_{m}^{r}}
$$

ДокАЗАТЕЛЬство. Домножим обе части неравенства $(35)$ на $e^{-\tau\left(\frac{d}{2}-\frac{1}{\gamma-1}\right)}$ :

$$
\left\|e^{\tau \mathscr{L}} P_{-}^{n} f\right\|_{H_{m}^{r}} \leqslant C e^{-\left(\frac{n+1}{2}+\frac{d}{2}-\frac{1}{\gamma-1}\right) \tau}\|f\|_{H_{m}^{r}} .
$$

Выбрав $n$ настолько большим, чтобы выполнялось неравенство

$$
s \leqslant \frac{n+1}{2}+\frac{d}{2}-\frac{1}{\gamma-1},
$$

и $m$, удовлетворяющее условию следствия 3.1 , мы получаем искомую оценку.

СлеДСтвИЕ 3.3. Для произвольных челых $r \geqslant 0, m>d / 2$ uf $f \in H_{m}^{r}$ справедлива оченка

$$
\left\|e^{\tau \mathscr{L}} f\right\|_{H_{m}^{r}} \leqslant C e^{-\tau\left(\frac{d}{2}-\frac{1}{\gamma-1}\right)}\|f\|_{H_{m}^{r}} .
$$


ДОКАЗАТЕЛЬСТВО. Положим в условиях следствия $3.1 n=-1$ и домножим обе части неравенства $(35)$ на $e^{-\tau\left(\frac{d}{2}-\frac{1}{\gamma-1}\right)}$. Тогда, так как $P_{-}^{n}=I$ при $n<0$, получим:

$$
\left\|e^{\tau \mathscr{L}} f\right\|_{H_{m}^{r}} \leqslant C e^{-\tau\left(\frac{d}{2}-\frac{1}{\gamma-1}\right)}\|f\|_{H_{m}^{r}} .
$$

ЗАМЕчАНИЕ. Из следствия 3.3 следует, что при условии $\gamma>1+2 / d$, следуюшем из (18), решение линейной задачи Коши (25) стремится к нулю с экспоненциальной (в $\tau$-представлении) скоростью сходимости.

ДОКАЗАТЕЛЬСТВО ТЕОРЕМЫ 3.2. Из следствИя 3.1 вЫтекает, что дЛя Любого $\varepsilon>0$ полугруппа $e^{\frac{\tau}{2}\left(-\frac{d}{2}+m-\varepsilon\right)} e^{\tau \Lambda}$ является равномерно ограниченной полугруппой в пространстве $P_{-}^{n} H_{m}^{r}$. Тогда из теоремы Хилле-Иосида о резольвенте производящего оператора следует $\sigma\left(\Lambda P_{-}^{n}\right) \subset\{\lambda \in \mathbb{C}: \operatorname{Re} \lambda \leqslant d / 4-m / 2\} \cup\{0\}$ (см., например, [25]). Это включение вместе с (30) дает равенство

$$
\sigma\left(\Lambda P_{-}^{n}\right)=\left\{\lambda \in \mathbb{C}: \operatorname{Re} \lambda \leqslant \frac{d}{4}-\frac{m}{2}\right\} \cup\{0\}
$$

Утверждения теоремы 3.2 следуют из леммы 3.3 с учетом (29) и последнего равенства. Теорема 3.2 , а следовательно, и теорема 3.1 доказаны.

Итак, спектр оператора $\mathscr{L}$ обладает тем свойством, что за счет выбора достаточно большого значения $m$ мы можем отодвинуть непрерывную часть спектра сколь угодно далеко влево от начала координат, выделяя все большее количество собственных значений дискретной части спектра (см. рис. 1). Эта особенность позволит нам в $\S 5$ построить инвариантные многообразия для нелинейного уравнения (23).

\section{$\S 4$. Существование и единственность решения нелинейной задачи Коши. Полугруппа $S^{\tau}$}

В этом параграфе мы докажем локальное сушествование и единственность решения $\widehat{z}(\tau, \xi)$ задачи Коши (23), (24) на всей временной оси $\tau \in \mathbb{R}_{+}$.

Схематично, процесс доказательства будет состоять в следующем. Зафиксируем некоторый момент времени $T>0$, достаточно малый параметр $\varepsilon>0$ и разобьем временную ось $\mathbb{R}_{+}$на отрезки $\left\{\mathscr{R}_{i}\right\}, i \in \mathbb{N} \cup\{0\}$ :

$$
\mathbb{R}_{+}=\bigcup_{i=0}^{\mathbb{N}} \mathscr{R}_{i}
$$

где $\mathscr{R}_{0}=[0, T], \mathscr{R}_{1}=[T-\varepsilon, 2 T], \mathscr{R}_{2}=[2 T-\varepsilon, 3 T], \ldots, \mathscr{R}_{k}=[k T-\varepsilon,(k+1) T]$, $k \in \mathbb{N}$.

После этого докажем сушествование решения $\widehat{z}(\tau, \xi)$ задачи Коши на интервале $\tau \in[0, T]$. Решая новую задачу Коши на интервале $\mathscr{R}_{1}$ с начальным условием $\widehat{z}(T-\varepsilon, \xi)$, полученным на предыдушем этапе, мы получаем продолжение решения на следуюший отрезок $\mathscr{R}_{1}$. Итеративно продолжая этот процесс на последующие 
отрезки $\mathscr{R}_{i}$, мы получим искомое решение задачи Коши. Ввиду наложенности отрезков $\mathscr{R}_{i}$ полученная функция $\widehat{z}(\tau, \xi)$ будет решением задачи Коши на всей временной оси $\mathbb{R}_{+}$.

Сушествование решения $\widehat{z}(\tau, \xi)$ на отрезке $[0, T]$ вьводится из теоремы о неявной функции. Для применимости этой теоремы мы введем следующие пространства, в которых будем рассматривать нашу задачу.

Пусть $T$ - некоторый фиксированный момент времени. Определим пространство

$$
\begin{gathered}
L_{2}\left(H_{m}^{r}\right)=L_{2}\left(H_{m}^{r}\left(\mathbb{R}^{d}\right)\right)=L_{2}\left(0, T ; H_{m}^{r}\left(\mathbb{R}^{d}\right)\right) \cap C\left([0, T] ; H_{m}^{r}\left(\mathbb{R}^{d}\right)\right) \\
\|\cdot\|_{L_{2}\left(H_{m}^{r}\right)}=\max \left(\|\cdot\|_{L_{2}\left(0, T ; H_{m}^{r}\left(\mathbb{R}^{d}\right)\right)},\|\cdot\|_{L_{\infty}\left(0, T ; H_{m}^{r}\left(\mathbb{R}^{d}\right)\right)}\right) .
\end{gathered}
$$

ЛЕмма 4.1. Пусть заданы иелье $r, d / 2<r<\gamma, u m>0$. Отобрахсение $F(\widehat{z})=|\widehat{z}|^{\gamma-1} \widehat{z}: L_{2}\left(H_{m}^{r}\right) \rightarrow L_{2}\left(H_{m}^{r}\right)$ является отображением класса $C^{2}$, т.е. дважсды непрерывно дифферениируемо на пространстве $L_{2}\left(H_{m}^{r}\right)$.

ДоказАтельство. Покажем, что $F$ отображает $L_{2}\left(H_{m}^{r}\right)$ в $L_{2}\left(H_{m}^{r}\right)$. При $r>d / 2$ из теоремы вложения Соболева следует неравенство

$$
\|\widehat{z}(\tau, \cdot)\|_{C_{0}\left(\mathbb{R}^{d}\right)} \leqslant \widetilde{C}\|\widehat{z}(\tau, \cdot)\|_{H_{0}^{r}\left(\mathbb{R}^{d}\right)}
$$

с некоторой константой $\widetilde{C}>0$. Поэтому справедливы следующие оценки:

$$
\begin{aligned}
& \left\|\left(1+|\xi|^{2}\right)^{m / 2}\left|\partial^{\alpha}\left(\widehat{z}(\tau, \cdot)^{\gamma-1} \widehat{z}(\tau, \cdot)\right)\right|\right\|_{L_{2}\left(\mathbb{R}^{d}\right)} \\
& \quad \leqslant C \sum_{|\beta| \leqslant|\alpha|}\left\||\widehat{z}(\tau, \cdot)|^{\gamma-|\beta|}\right\|_{C_{0}\left(\mathbb{R}^{d}\right)}\|\widehat{z}(\tau, \cdot)\|_{H_{m}^{r}\left(\mathbb{R}^{d}\right)} \\
& \quad \leqslant C_{1} \sum_{|\beta| \leqslant|\alpha|}\left(1+\|\widehat{z}(\tau, \cdot)\|_{H_{m}^{r}\left(\mathbb{R}^{d}\right)}^{\gamma-|\beta|}\right)\|\widehat{z}(\tau, \cdot)\|_{H_{m}^{r}\left(\mathbb{R}^{d}\right)}<\infty, \quad|\alpha| \leqslant r .
\end{aligned}
$$

Аналогично можно показать, что производная $F^{\prime}(\widehat{z})=\gamma|\widehat{z}|^{\gamma-1}$ в точке $\widehat{z}$ также отображает $L_{2}\left(H_{m}^{r}\right)$ в $L_{2}\left(H_{m}^{r}\right)$. Из условия $(18)$ на $\gamma$ непосредственно вытекает, что $\gamma>2$. Это, в свою очередь, влечет существование непрерывной второй производной $F^{\prime \prime}$ у функции $F$.

Уравнению (23) сопоставим уравнение в вариациях

$$
\partial_{\tau} \widehat{z}=\mathscr{L} \widehat{z}+h, \quad h \in L_{2}\left(H_{m}^{r}\right), \quad(\tau, \xi) \in(0, \infty) \times \mathbb{R}^{d}
$$

с некоторым начальным условием

$$
\left.\widehat{z}\right|_{\tau=0}=z_{0}(x)
$$

Введем оператор $Q: L_{2}\left(H_{m}^{r}\left(\mathbb{R}^{d}\right)\right) \times H_{m}^{r}\left(\mathbb{R}^{d}\right) \rightarrow L_{2}\left(H_{m}^{r}\left(\mathbb{R}^{d}\right)\right), Q\left(h, z_{0}\right)=\widehat{z}(\tau, \xi)$, сопоставляющий начальному условию $z_{0}$ и правой части $h$ решение уравнения в вариациях (39).

Определим норму на пространстве $L_{2}\left(H_{m}^{r}\right) \times H_{m}^{r}$ стандартным образом:

$$
\left\|\left(h, z_{0}\right)\right\|_{L_{2}\left(H_{m}^{r}\right) \times H_{m}^{r}}^{2}=\|h\|_{L_{2}\left(H_{m}^{r}\right)}^{2}+\left\|z_{0}\right\|_{H_{m}^{r}}^{2} .
$$


ЛЕмма 4.2. Для любых целых $r, d / 2<r<\gamma, m>d / 2, h \in L_{2}\left(H_{m}^{r}\right), z_{0} \in H_{m}^{r}$ существует и единственно решение $\widehat{z} \in L_{2}\left(H_{m}^{r}\right)$ задачи Коии (39), (40) и соответствующий разрешающий оператор $Q: L_{2}\left(H_{m}^{r}\right) \times H_{m}^{r} \rightarrow L_{2}\left(H_{m}^{r}\right)$, $Q\left(h, z_{0}\right)=\widehat{z}$, ограничен.

ДокАЗАТЕЛьство. Представим оператор $Q\left(h, z_{0}\right)$ в виде

$$
Q\left(h, z_{0}\right)=Q\left(0, z_{0}\right)+Q(h, 0)
$$

Непрерывность первого слагаемого в (41) вытекает из следствия 3.3:

$$
\left\|Q\left(0, z_{0}\right)\right\|_{L_{2}\left(H_{m}^{r}\right)} \leqslant C_{1}\left(\int_{0}^{T} e^{-2 \tau\left(\frac{d}{2}-\frac{1}{\gamma-1}\right)} d \tau\right)^{1 / 2}\left\|z_{0}\right\|_{H_{m}^{r}} \leqslant C_{2}\left\|z_{0}\right\|_{H_{m}^{r}}
$$

Докажем непрерывность второго слагаемого. Справедливы следующие оценки:

$$
\begin{aligned}
\|Q(h, 0)(\tau, \cdot)\|_{H_{m}^{r}} & =\left\|\int_{0}^{\tau} e^{\mathscr{L}\left(\tau-\tau^{\prime}\right)} h\left(\tau^{\prime}, \cdot\right) d \tau^{\prime}\right\|_{H_{m}^{r}} \\
& \leqslant \int_{0}^{\tau}\left\|e^{\mathscr{L}\left(\tau-\tau^{\prime}\right)} h\left(\tau^{\prime}, \cdot\right)\right\|_{H_{m}^{r}} d \tau^{\prime} \\
& \leqslant \int_{0}^{\tau} C e^{-\tau^{\prime}\left(\frac{d}{2}-\frac{1}{\gamma-1}\right)}\left\|h\left(\tau^{\prime}, \cdot\right)\right\|_{H_{m}^{r}} d \tau^{\prime} \leqslant C_{3}\|h\|_{L_{2}\left(H_{m}^{r}\right)}
\end{aligned}
$$

с некоторой константой $C_{3}$. Отсюда получаем, что с некоторой константой $C_{4}=$ $C_{4}(T)$ выполнена оценка

$$
\|Q(h, 0)\|_{L_{2}\left(H_{m}^{r}\right)} \leqslant C_{4}\|h\|_{L_{2}\left(H_{m}^{r}\right)}
$$

ЛЕмма 4.3. Для любъх иельх $r, d / 2<r<\gamma, m>d / 2$ и любого начального условия $z_{0} \in H_{m}^{r}\left(\mathbb{R}^{d}\right)$ из некоторой окрестности нуля $V_{0}$ существует единственное решение $\widehat{z}(\tau, \xi) \in L_{2}\left(H_{m}^{r}\right)$ задачи (23), (24), непрерывно зависящее от начального условия $z_{0}$. Причем отображсение $S: z_{0} \rightarrow \widehat{z}$ является отображением класса $C^{2}$. Более того, справедливо следующее соотношение:

$$
S^{\prime}(0) z_{0}=Q\left(0, z_{0}\right)
$$

ДоКАЗАТЕЛЬСТво. В обозначениях предыдущей леммы уравнение (23) эквивалентно следующему:

$$
\widehat{z}=Q\left(0, z_{0}\right)+Q\left(|\widehat{z}|^{\gamma-1} \widehat{z}, 0\right) .
$$

Введем оператор $\Phi\left(\widehat{z}, z_{0}\right): L_{2}\left(H_{r}^{m}\right) \times H_{m}^{r} \rightarrow L_{2}\left(H_{m}^{r}\right)$ как

$$
\Phi\left(\widehat{z}, z_{0}\right)=Q\left(0, z_{0}\right)+Q\left(|\widehat{z}|^{\gamma-1} \widehat{z}, 0\right)-\widehat{z} .
$$

Тогда уравнение (42) эквивалентно уравнению

$$
\Phi\left(\widehat{z}, z_{0}\right)=0
$$


Очевидно, что $\Phi(0,0)=0$. Более того, $\Phi\left(\widehat{z}, z_{0}\right)$ является отображением класса $C^{2}$. Действительно, $Q\left(0, z_{0}\right)$ линейно и непрерьвно по $z_{0}$ в силу леммы 4.2 . По леммам $4.1,4.2$ отображение $Q\left(|\widehat{z}|^{\gamma-1} \widehat{z}, 0\right)$ является отображением класса $C^{2}$, а следовательно, и $\Phi\left(\widehat{z}, z_{0}\right)$ является отображением класса $C^{2}$.

Далее, дифференциал $\Phi_{\widehat{z}}^{\prime}(0,0)$ совпадает с оператором $-I$, а следовательно, обратим. По теореме о неявной функции уравнение (43) имеет единственное решение в некоторой достаточно малой окрестности нуля $V_{0}$ в топологии пространства $L_{2}\left(H_{r}^{m}\right) \times H_{m}^{r}$ и отображение $S: z_{0} \rightarrow \widehat{z}$, сопоставляющее начальному условию $z_{0}$ решение $\widehat{z}$, является отображением класса $C^{2}$. Тогда по правилу дифференцирования неявной функции дифференциал $S^{\prime}(0) z_{0}$ удовлетворяет уравнению в вариациях $(39)$ с $h=0$, т.е. $S^{\prime}(0) z_{0}=Q\left(0, z_{0}\right)$.

Обозначим через $\gamma_{\tau}$ оператор сужения функций из $L_{2}\left(H_{m}^{r}\right)$ на пространство $H_{m}^{r}$ :

$$
\gamma_{\tau} \widehat{z}(\cdot, \cdot)=\widehat{z}(\tau, \cdot)
$$

Оператор $\gamma_{\tau}$ определен и непрерывен в $L_{2}\left(H_{m}^{r}\right)$ ввиду того, что по определению $L_{2}\left(H_{m}^{r}\right) \subset C\left([0, T] ; H_{m}^{r}\right)$.

Сопоставим уравнению (23) полугруппу $\left\{S^{\tau}\right\}_{\tau \in[0, T]}: H_{m}^{r} \rightarrow H_{m}^{r}$ по формуле $S^{\tau} z_{0}=\gamma_{\tau} S\left(z_{0}\right)$, где $S$ - разрешаюший оператор, построенный в предыдушей лемме. Эта полугруппа определена в достаточно малой окрестности нуля $V_{0} \subset H_{m}^{r}$.

Лемма 4.4. Полугруппа $\left\{S^{\tau}\right\}_{\tau \in[0, T]}$ является отображением класса $C^{2}$, и ее производная определяется следующей формулой:

$$
\left(S^{\tau}\right)_{z_{0}}^{\prime}(0)=e^{\tau \mathscr{L}}
$$

ДокАЗАТЕЛьство. Поскольку оператор $S$ является отображением класса $C^{2}$, то оператор $S^{\tau}$ как суперпозиция оператора $S$ и непрерывного линейного оператора $\gamma_{\tau}$ также будет отображением класса $C^{2}$. Из определения оператора $Q$ следует, что

$$
\gamma_{\tau} Q\left(0, z_{0}\right)=e^{\tau \mathscr{L}} z_{0}
$$

где $e^{\tau \mathscr{L}}$ - разрешаюший оператор задачи Коши (25).

Тогда

$$
\left(S^{\tau}\right)_{z_{0}}^{\prime}=\gamma_{\tau} S^{\prime}\left(z_{0}\right)=\gamma_{\tau} Q\left(0, z_{0}\right)=e^{\tau \mathscr{L}} z_{0}
$$

Теорема 4.1. Для любых цельи $r, d / 2<r<\gamma, m>d / 2$ и произвольного начального условия $z_{0} \in H_{m}^{r}\left(\mathbb{R}^{d}\right)$ из некоторой окрестности нуля $V_{0}$ существует единственное решение $\widehat{z}(\tau, \xi) \in L_{2}\left(0, \infty ; H_{m}^{r}\right) \cap C\left([0, \infty) ; H_{m}^{r}\right)$ задачи (23), (24), непрерывно зависящее от начального условия $z_{0}$.

При этом соответствующая разрешающая полугруппа $\left\{S^{\tau}\right\}_{\tau>0}: H_{m}^{r} \rightarrow$ $H_{m}^{r}$ является отображением класса $C^{2}$ и ее производная в нуле находится из соотношения

$$
S^{\prime}(0) z_{0}=Q\left(0, z_{0}\right) .
$$


ДоКАЗАТЕЛьство. Пользуясь следствием 3.3 , зададимся моментом времени $T$ таким, чтобы выполнялось неравенство

$$
\left\|e^{T \mathscr{L}_{2}}\right\|_{H_{m}^{r}} \leqslant \frac{1}{4}\left\|z_{0}\right\|_{H_{m}^{r}}
$$

для всех $z_{0} \in H_{m}^{r}$.

Пусть $\widehat{z}(\tau, \xi)$ - решение задачи Коши (23), (24), определенное для $\tau \in[0, T]$. Из (42) следуют оценки:

$$
\begin{aligned}
\left\|S^{T} z_{0}\right\|_{H_{m}^{r}} & =\|\widehat{z}(T, \cdot)\|_{H_{m}^{r}} \leqslant\left\|\gamma_{T} Q\left(0, z_{0}\right)\right\|_{H_{m}^{r}}+\left\|\gamma_{T} Q\left(|\widehat{z}|^{\gamma-1} \widehat{z}, 0\right)\right\|_{H_{m}^{r}} \\
& \leqslant\left(\frac{1}{4}+\widetilde{C}\|\widehat{z}\|_{L_{2}\left(H_{m}^{r}\right)}^{\gamma-1}\right)\left\|z_{0}\right\|_{H_{m}^{r}}
\end{aligned}
$$

В последнем неравенстве мы воспользовались непрерьвностью операторов $\gamma_{T}, Q$ и $S$. Тогда при $z_{0}$ из достаточно малой окрестности нуля $V_{0}$ ввиду непрерывной зависимости решения от начальных данных будет выполнено

$$
\left\|S^{T} z_{0}\right\|_{H_{m}^{r}} \leqslant \frac{1}{2}\left\|z_{0}\right\|_{H_{m}^{r}}
$$

т.е. значение решения $\widehat{z}(\tau, \xi)$ в момент времени $T$ будет снова принадлежать окрестности $V_{0}$. Это решение будет принадлежать пространству $L_{2}\left(0, T ; H_{m}^{r}\left(\mathbb{R}^{d}\right)\right) \cap$ $C\left([0, T] ; H_{m}^{r}\left(\mathbb{R}^{d}\right)\right)$.

Зададимся малым $\varepsilon>0$. Решая задачу Коши на отрезке $\mathscr{R}_{1}=[T-\varepsilon, 2 T]$ с начальным условием $z(T-\varepsilon, \cdot)$, которое будет принадлежать окрестности $V_{0}$ при малых $\varepsilon$, мы получаем продолжение решения $z(\tau, \xi)$ на отрезок $[T, 2 T]$. Аналогичным образом, решая задачу Коши на отрезке $\mathscr{R}_{2}=[2 T-\varepsilon, 3 T]$ с начальным условием, полученным на предыдушем этапе, мы получаем продолжение решения на отрезок $[2 T, 3 T]$. Повторяя этот процесс, мы получаем решение $\widehat{z}(\tau, \xi)$, определенное на всей временной оси, принадлежашее пространству $L_{2}\left(0, \infty ; H_{m}^{r}\right) \cap C\left([0, \infty) ; H_{m}^{r}\right)$.

\section{§5. Инвариантные многообразия}

В этом параграфе мы докажем сушествование инвариантных относительно действия полугруппы $\left\{S^{\tau}\right\}_{\tau} \geqslant 0$ многообразий. Основой этого построения послужат инвариантные подпространства линейной полугрупшы $e^{\tau \mathscr{L}}$. Как мы увидим, полученные многообразия будут касательными в нуле к соответствующим инвариантным подпространствам линейной полугруппы $e^{\tau \mathscr{L}}$.

ЛЕмма 5.1. Пусть заданы иелье $r \geqslant 0,0 \leqslant n<m$. Подпространства $E_{+}^{n}=P_{+}^{n} H_{m}^{r}$ и $E_{-}^{n}=P_{-}^{n} H_{m}^{r}$ инвариантнь относительно действия полугруп$n b l e^{\tau \mathscr{L}}$. 
ДокАЗАТЕЛЬство. Действительно, $E_{+}^{n}$ является линейной оболочкой собственных векторов оператора $\mathscr{L}$ и, следовательно, инвариантно относительно действия порождаемой им полугрупшы. Докажем инвариантность $E_{-}^{n}$.

Для любой функции $g \in L_{2}\left(\mathbb{R}^{d}\right)$ из условия ортогональности

$$
\int_{\mathbb{R}^{d}} f(\xi) \xi^{\alpha} d \xi=0, \quad|\alpha| \leqslant n
$$

следует, что свертка $g * f$ удовлетворяет тому же условию ортогональности:

$$
\int_{\mathbb{R}^{d}}(g * f)(\xi) \xi^{\alpha} d \xi=\int_{\mathbb{R}^{d}} g\left(\xi^{\prime}\right) \int_{\mathbb{R}^{d}} f\left(\xi-\xi^{\prime}\right) \xi^{\alpha} d \xi d \xi^{\prime}=0, \quad|\alpha| \leqslant n .
$$

Так как действие полугруппы $e^{\tau \mathscr{L}}$ на вектор $f \in E_{-}^{n}$ в силу (31) есть свертка ядра $\frac{e^{\frac{\tau}{\gamma-1}}}{(4 \pi a(\tau))^{\frac{d}{2}}} e^{-\frac{|\xi|^{2}}{4 a(\tau)}}$ с функцией $f\left(\xi e^{\frac{\tau}{2}}\right)$, будет выполнено

$$
\int_{\mathbb{R}^{d}} e^{\tau \mathscr{L}} f(\xi) \xi^{\alpha} d \xi=0, \quad|\alpha| \leqslant n
$$

откуда следует, что $e^{\tau \mathscr{L}} f \in E_{-}^{n}$.

Теперь перейдем к построению инвариантных многообразий для нелинейной полугруппы $\left\{S^{\tau}\right\}_{\tau} \geqslant 0$. Дальнейшее исследование предварим основными фактами из теории инвариантных многообразий полугрупп (более подробное рассмотрение можно найти в книге А. В. Бабина, М. И. Вишика [26]).

Пусть $E$ - некоторое банахово пространство с нормой $\|\cdot\|_{E}, \mathscr{L}(E, E)-$ пространство линейных непрерывных отображений из $E$ в $E$ с операторной нормой $\|\cdot\|_{\mathscr{L}(E, E)} \cdot$

ОПРЕДЕЛЕНИЕ 5.1. Полугруппа линейных ограниченных операторов

$$
\left\{L^{\tau}\right\}_{\tau \in[0, \infty)}: E \rightarrow E
$$

называется почти устойчивой, если выполнены условия:

1) $L^{\tau}$ ограничены в $E$ по норме при $0 \leqslant \tau<\infty$ константой $C$;

2) $\left\langle L^{\tau} u, \varphi\right\rangle$ непрерывно по $\tau$ для любых $u \in E, \varphi \in \Phi^{*}$, где $\Phi^{*}$ - всюду плотное множество $E^{*}$;

3) существует $\rho_{0} \subset(0,1)$ такое, что вне круга $|\zeta| \leqslant \rho_{0}$ имеется лишш конечное множество $\sigma_{+}=\zeta_{1}, \ldots, \zeta_{N}$ точек спектра оператора $\left.L_{\tau}\right|_{\tau=1}$;

4) инвариантное подпространство оператора $\left.L_{\tau}\right|_{\tau=1}$, соответствующее $\sigma_{+}$, конечномерно.

Пусть задана полугруппа нелинейных операторов $S^{\tau}: E \rightarrow E, S^{\tau}(0)=0$, и пусть оператор $S=\left.S^{\tau}\right|_{\tau=1}$ допускает следующее представление:

$$
S u=L u+D(u),
$$

где $L$ - линейньй оператор, $D$ - малый по норме нелинейный оператор. Пусть $L$ обладает двумя инвариантньми подпространствами $E_{+}$и $E_{-}, E_{+} \cap E_{-}=\{0\}$, 
$E=E_{+}+E_{-}, L E_{+} \subset E_{+}, L E_{-} \subset E_{-}, \operatorname{dim} E_{+}<\infty$. Проекции элемента $u$ на подпространства $E_{+}$и $E_{-}$обозначим через $u_{+}$и $u_{-}$, а операторы проектирования через $P_{+}$и $P_{-}$соответственно:

$$
P_{+} u=u_{+}, \quad P_{-} u=u_{-}, \quad P_{+}+P_{-}=I .
$$

Норму элемента $u=u_{+}+u_{-}$переопределим как $\|u\|=\max \left(\left\|u_{+}\right\|,\left\|u_{-}\right\|\right)$, эта норма в силу теоремы Банаха будет эквивалентна исходной.

Сужение оператора $L$ на подпространства $E_{+}$и $E_{-}$обозначим через $L_{+}$и $L_{-}$ соответственно.

Укажем условия на операторы $L_{+}, L_{-}$и $D$, которые будем предполагать выполненными.

УСловиЕ 5.1 (об отделимости спектров операторов $L_{-}$и $L_{+}$). Сушествует константа $\rho>0$ и числа $\varepsilon_{+}>0, \varepsilon_{-}>0$ такие, что

$$
\left\|L_{-}\right\|_{\mathscr{L}(E, E)} \leqslant \rho\left(1-\varepsilon_{-}\right), \quad\left\|L_{+}^{-1}\right\|_{\mathscr{L}(E, E)} \leqslant \rho^{-1}\left(1-\varepsilon_{+}\right) .
$$

Смысл этого условия заключается в отделимости спектров операторов $L_{-}$и $L_{+}$ окружностью $|\zeta|=\rho$.

УСловиЕ 5.2 (о гладкости нелинейного члена). Существует достаточно малое $\nu$ такое, что выполнено

$$
D(0)=0, \quad D^{\prime}(0)=0, \quad\left\|D^{\prime}(u)\right\|_{\mathscr{L}(E, E)} \leqslant \nu, \quad u \in E .
$$

УСловиЕ 5.3 (о гёльдеровости оператора $D$ с показателем $\mu, 0<\mu \leqslant 1$ ).

$$
\begin{aligned}
\left\|D^{\prime}\right\|_{\mu} & \leqslant C_{0}, \\
\left\|D^{\prime}\right\|_{\mu} & =\sup _{0 \leqslant\|w-v\| \leqslant 1} \frac{\left\|D^{\prime}(w)-D^{\prime}(v)\right\|_{\mathscr{L}(E, E)}}{\|w-v\|_{E}^{\mu}} .
\end{aligned}
$$

Если отображение $D$ удовлетворяет условию 5.3 , то будем говорить, что $D$ является отображением класса $C^{1+\mu}$.

Мы будем искать инвариантные многообразия $M_{+}$и $M_{-}$, касательные в нуле к пространствам $E_{+}$и $E_{-}$, в виде графиков функций $g_{+}: V_{0} \subset E_{+} \rightarrow E_{-}$и $g_{-}: V_{0}^{\prime} \subset$ $E_{-} \rightarrow E_{+}$соответственно:

$$
\begin{aligned}
& M_{+}=\left\{u \in E: u=u_{+}+g_{+}\left(u_{+}\right), u_{+} \in V_{0}\right\}, \\
& M_{-}=\left\{u \in E: u=u_{-}+g_{-}\left(u_{-}\right), u_{-} \in V_{0}^{\prime}\right\},
\end{aligned}
$$

где $V_{0}$ и $V_{0}^{\prime}$ - достаточно малые окрестности нуля в топологии подпространств $E_{+}$ и $E_{-}$(см. рис. 2). Здесь функции $g_{+}, g_{-}$рассматриваются в классе функций $C^{1+\mu}$, удовлетворяющих условиям:

$$
g_{ \pm}(0)=0, \quad g_{ \pm}^{\prime}(0)=0, \quad\left\|g_{ \pm}^{\prime}(u)\right\|_{\mu} \leqslant q, \quad u \in E
$$

с некоторым $q>0$. 


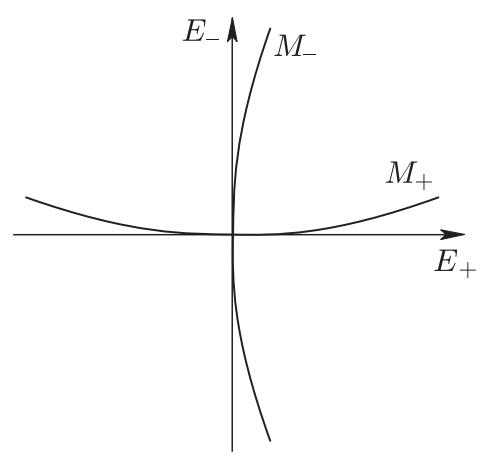

Рис. 2

TEорема 5.1 (о сушествовании инвариантного многообразия). Пусть $S^{\tau}: E \rightarrow$ $E, S^{\tau}(0)=0,-$ полугруппа класса $C^{1+\mu}$, и пусть выполнены все наложсенные выше условия на операторы $S=\left.S^{\tau}\right|_{\tau=1}, L, L_{+}, L_{-}, D$. Кроме того, пусть существует такое $\varepsilon>0$, что при $0 \leqslant t \leqslant \varepsilon$ операторы $D^{\tau}=S^{\tau}-\left(S^{\tau}\right)^{\prime}(0)$

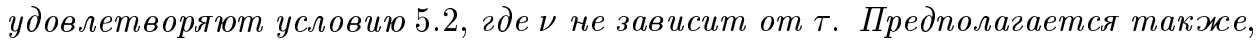
что $\left(S^{\tau}\right)^{\prime}(0)$ образуют почти устойчивую полугруппу с $\rho_{0}<\rho$, где $\rho$ - такое же, как и в условии 5.1, и пусть выполнена оценка

$$
\left\|S^{\tau} u\right\|_{E} \leqslant C\|u\|_{E}, \quad \tau \in[0,1] .
$$

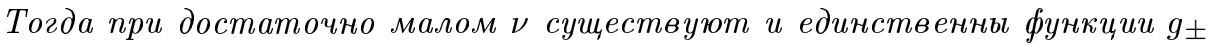
класса $C^{1+\mu}$ такие, что многообразия $M_{+}$и $M_{-}$инвариантны относительно $S^{\tau}: S^{\tau} M_{ \pm} \subset M_{ \pm}$. Причем эти многообразия являются касательными в нуле $\kappa$ подпространствам $E_{+}$и $E_{-}$соответственно, т.е. $g_{ \pm}^{\prime}(0)=0$.

При этом для $u \in M_{-}$справедлива следующая оченка:

$$
\left\|S^{\tau} u\right\|_{E} \leqslant C \rho^{\tau}\|u\|_{E}, \quad \tau>0, \quad u \in M_{-} .
$$

Доказательство см. в [26].

Пусть $\varphi \in C_{0}^{\infty}(\mathbb{R}), \varphi=1$ при $|x| \leqslant 1 / 2, \varphi(x)=0$ при $|x|>1$ и $0 \leqslant \varphi \leqslant 1$, $0 \leqslant\left|\varphi^{\prime}\right| \leqslant 1$. Положим

$$
\Psi_{\varepsilon}(\widehat{z})=\varphi\left(\frac{\|\widehat{z}\|_{H_{m}^{r}}^{3}}{\varepsilon}\right)
$$

Вместо уравнения (23) рассмотрим уравнение с нелинейностью вида $F_{\varepsilon}(\widehat{z})=$ $|\widehat{z}|^{\gamma-1} \widehat{z} \Psi_{\varepsilon}(\widehat{z}):$

$$
\frac{\partial \widehat{z}(\tau, \xi)}{\partial \tau}=\Delta \widehat{z}+\frac{1}{2}(\xi, \nabla) \widehat{z}+\frac{1}{\gamma-1} \widehat{z}+|\widehat{z}|^{\gamma-1} \widehat{z} \Psi_{\varepsilon}(\widehat{z}) .
$$

Лемма 5.2. Отображсние $F_{\varepsilon}(\widehat{z})=|\widehat{z}|^{\gamma-1} \widehat{z} \Psi_{\varepsilon}(\widehat{z}): H_{m}^{r} \rightarrow H_{m}^{r}$ является отображением класса $C^{2}$, и для достаточно мальх $\varepsilon>0$ справедлива оченка

$$
\left\|F_{\varepsilon}^{\prime}(\widehat{z})\right\|_{\mathscr{L}\left(H_{m}^{r}, H_{m}^{r}\right)} \leqslant C \varepsilon
$$

әде С не зависит от $\widehat{z} и \varepsilon$. 
ДокАЗАТЕЛЬСтво. Утверждение леммы о том, что $F_{\varepsilon}$ является отображением класса $C^{2}$, непосредственно следует из леммы 4.1. Действительно, поскольку отображения $\|\widehat{z}\|_{H_{m}^{r}}^{3}, \varphi$ и $|\widehat{z}|^{\gamma-1} \widehat{z}$ являются отображениями класса $C^{2}$, то и $F_{\varepsilon}$ будет принадлежать тому же классу.

Оценим норму производной оператора $F_{\varepsilon}$. Заметим, что $F_{\varepsilon}(\widehat{z})=0$ для $\widehat{z}$ такого, что $\|\widehat{z}\|_{H_{m}^{r}}>\sqrt[3]{\varepsilon}$. Поэтому будем предполагать, что $\|\widehat{z}\|_{H_{m}^{r}} \leqslant \sqrt[3]{\varepsilon}$. Аналогично оценкам, приводимым при доказательстве леммы 4.1 , используя свойства функции $\varphi$, с учетом того, что

$$
\Psi_{\varepsilon}(\widehat{z}) \leqslant 1, \quad\left|\Psi_{\varepsilon}^{\prime}(\widehat{z})\right| \leqslant \frac{C_{0}}{\varepsilon}\|\widehat{z}\|_{H_{m}^{r}}^{2}
$$

для достаточно малых $\varepsilon$ можно получить оценки

$$
\begin{aligned}
\left\|F_{\varepsilon}^{\prime}(\widehat{z})\right\|_{\mathscr{L}\left(H_{m}^{r}, H_{m}^{r}\right)} & \leqslant C_{1}\left\||\widehat{z}|^{\gamma-1}\right\|_{H_{m}^{r}}+C_{2} \frac{1}{\varepsilon}\left\||\widehat{z}|^{\gamma-1} \widehat{z}\right\|_{H_{m}^{r}} \cdot\|\widehat{z}\|_{H_{m}^{r}}^{2} \\
& \leqslant C_{3}\|\widehat{z}\|_{H_{m}^{r}}+C_{4} \frac{1}{\varepsilon}\|\widehat{z}\|_{H_{m}^{r}}^{4} \leqslant C \varepsilon .
\end{aligned}
$$

Отметим, что теорема 4.1 остается верной и в том случае, когда мы рассматриваем уравнение (48) вместо уравнения (23). Также соответствуюшая разрешающая полугруппа $\left\{S_{\varepsilon}^{\tau}\right\}_{\tau}>0$ принадлежит классу $C^{2}$, и очевидно, что в достаточно малой окрестности нуля полугруппа $\left\{S_{\varepsilon}^{\tau}\right\}_{\tau>0}$ совпадает с полугруппой $\left\{S^{\tau}\right\}_{\tau>0}$.

Зададимся некоторьм $s>0$, не являюшимся точкой спектра оператора $-\mathscr{L}$, и возьмем достаточно большие $m$ и $n$, как в условиях следствия $3.2, r \in \mathbb{N}, d / 2<$ $r<\gamma$. Проверим вьполнение условий теоремы 5.1 в пространстве $E=H_{m}^{r}\left(\mathbb{R}^{d}\right)$.

В нашем случае $\left\{S_{\varepsilon}^{\tau}\right\}_{\tau>0}$ - разрешаюшая полугруппа уравнения (48), $S_{\varepsilon}=$ $\left.S_{\varepsilon}^{\tau}\right|_{\tau=1}, L=e^{\mathscr{L}}, L_{+}=L P_{+}^{n}, L_{-}=L P_{-}^{n}, E_{+}=E_{+}^{n} \equiv P_{+}^{n} E, E_{-}=E_{-}^{n} \equiv P_{-}^{n} E$, $\rho=e^{-s}$.

Выполнение условия 5.1 обеспечивается следствием 3.2, если вместо параметpa $s$ взять параметр $s+\sigma$, где $\sigma>0$ достаточно мало.

Нелинейный член $D_{\varepsilon}$ в нашем случае определим как

$$
D_{\varepsilon}\left(z_{0}\right)=S_{\varepsilon}\left(z_{0}\right)-S_{\varepsilon}^{\prime}(0) z_{0}=S_{\varepsilon}\left(z_{0}\right)-e^{\mathscr{L}} z_{0} .
$$

Из теоремы 4.1 следует, что $D_{\varepsilon}$ является отображением класса $C^{2}$, а следовательно, и класса $C^{1+\mu}$ с любым $\mu \in(0,1]$.

Докажем выполнение условия 5.2. Равенство $D_{\varepsilon}(0)=D_{\varepsilon}^{\prime}(0)=0$ следует непосредственно из определения нелинейного члена $D_{\varepsilon}$.

Лемма 5.3. Семейство операторов $D_{\varepsilon}^{\tau}=S_{\varepsilon}^{\tau}-\left(S_{\varepsilon}^{\tau}\right)^{\prime}(0), 0 \leqslant \tau \leqslant \delta, \delta>0$, удовлетворяет оценке

$$
\left\|\left(D_{\varepsilon}^{\tau}\left(z_{0}\right)\right)^{\prime}\right\|_{\mathscr{L}(E, E)} \leqslant C(\varepsilon)
$$

где $C(\varepsilon) \rightarrow 0$ при $\varepsilon \rightarrow 0$. 
ДокАЗАТЕЛьство. Справедливо представление

$$
S_{\varepsilon}^{\tau} z_{0}=\left(S_{\varepsilon}^{\tau}\right)^{\prime}(0) z_{0}+\gamma_{\tau} Q\left(|\widehat{z}|^{\gamma-1} \widehat{z} \Psi_{\varepsilon}(\widehat{z}), 0\right)=e^{\tau \mathscr{L}} z_{0}+\gamma_{\tau} Q\left(|\widehat{z}|^{\gamma-1} \widehat{z} \Psi_{\varepsilon}(\widehat{z}), 0\right),
$$

где в прежних обозначениях $Q$ - разрешающий оператор уравнения в вариациях $(39), \gamma_{\tau}$ - оператор сужения. Тогда ввиду непрерывности операторов $\gamma_{\tau}, Q$ и непрерывной зависимости решения от начальных данных из леммы 5.2 следует искомая оценка с некоторой функцией $C(\varepsilon)$, стремящейся к нулю при $\varepsilon \rightarrow 0$ :

$$
\begin{aligned}
\left\|\left(D_{\varepsilon}^{\tau}\left(z_{0}\right)\right)^{\prime}\right\|_{\mathscr{L}(E, E)} & =\left\|\left(\gamma_{\tau} Q\left(|\widehat{z}|^{\gamma-1} \widehat{z} \Psi_{\varepsilon}(\widehat{z}), 0\right)\right)^{\prime}\right\|_{\mathscr{L}(E, E)} \\
& =\left\|\left(\gamma_{\tau} Q\left(F_{\varepsilon}(\widehat{z}), 0\right)\right)^{\prime}\right\|_{\mathscr{L}(E, E)} \leqslant C(\varepsilon) .
\end{aligned}
$$

В частности, утверждение леммы справедливо и для оператора $D_{\varepsilon}=\left.D_{\varepsilon}^{\tau}\right|_{\tau=1}$. Отсюда получаем выполнение условия 5.2. Условие 5.3 следует из принадлежности полугруппы $\left\{S_{\varepsilon}^{\tau}\right\}_{\tau}>0$ классу $C^{2}$.

Свойство почти устойчивости полугрупшы $\left(S_{\varepsilon}^{\tau}\right)^{\prime}(0)$ и оценка $(47)$ следуют из теоремы 3.1 и следствия 3.3.

Итак, ввиду того что полугруппы $\left(S_{\varepsilon}^{\tau}\right)$ и $\left(S^{\tau}\right)$ совпадают в достаточно малой окрестности нуля, из теоремы 5.1 следует

Теорема 5.2. Пусть заданы $s>0, r \in \mathbb{N}, d / 2<r<\gamma$. Тогда для достаточно больиих челых чисел $n=n(s), m=m(s)$ в некоторой окрестности $\mathscr{W}_{0}$ нуля в пространстве $H_{m}^{r}\left(\mathbb{R}^{d}\right)$ существуют инвариантные относительно полугруппы $S^{\tau}$ многообразия $M_{+}^{s}, M_{-}^{s}$ вида

$$
\begin{aligned}
& M_{+}^{s}=\left\{y \in H_{m}^{r}: y=y_{+}+g_{+}\left(y_{+}\right), y_{+} \in V_{0} \equiv \mathscr{W}_{0} \cap E_{+}^{n} \subset E_{+}^{n}\right\}, \\
& M_{-}^{s}=\left\{y \in H_{m}^{r}: y=y_{-}+g_{-}\left(y_{-}\right), y_{-} \in V_{0}^{\prime} \equiv \mathscr{W}_{0} \cap E_{-}^{n} \subset E_{-}^{n}\right\},
\end{aligned}
$$

где $g_{ \pm}$- отображения из $E_{ \pm}^{n}$ в $E_{\mp}^{n}$ класса $C^{1+\mu}, 0<\mu \leqslant 1$, определеннье в окрестностя $V_{0}$ и $V_{0}^{\prime}$ соответственно и удовлетворяющие условиям $g(0)=$ $g^{\prime}(0)=0$.

При этом для әлементов $z_{0} \in M_{-}(s)$ верна следующая оценка:

$$
\left\|S^{\tau} z_{0}\right\|_{H_{m}^{r}\left(\mathbb{R}^{d}\right)} \leqslant C e^{-s \tau}\left\|z_{0}\right\|_{H_{m}^{r}\left(\mathbb{R}^{d}\right)}
$$

\section{§6. Доказательство стабилизации полулинейного уравнения}

Итак, мы построили инвариантное многообразие $M_{-}^{s}$ такое, что решение нелинейной задачи Коши с начальным условием, лежашим на этом многообразии, стабилизируется к нулю как $e^{-s \tau}$ в $\tau$-представлении или, возврашаясь к исходным переменньг $(x, t)$, как $1 / t^{s}$. Для доказательства теоремы 2.1 нам необходимо построить оператор продолжения начальных условий, определенных на $\Omega$, на пространство $\mathbb{R}^{d}$ таким образом, чтобы продолженное решение лежало на инвариантном многообразии.

ТеОРема 6.1. Пусть $r, m u s$ - такие жсе, как в теореме 5.2. Тогда существует оператор $R_{s}$, продолэсающий функиии из $\Omega$ в $\mathbb{R}^{d}$, которьй непрерывно отображает некоторую достаточно малую окрестность нуля $\mathscr{V}_{0} \subset H_{m}^{r}(\Omega)$ в $M_{-}^{s} \subset H_{m}^{r}\left(\mathbb{R}^{d}\right):$

$$
\left\|R_{s}\left(y_{0}(\cdot)\right)\right\|_{H_{m}^{r}\left(\mathbb{R}^{d}\right)} \leqslant C\left\|y_{0}(\cdot)\right\|_{H_{m}^{r}(\Omega)}, \quad y_{0} \in \mathscr{V}_{0}
$$


ДокАЗАТЕЛЬСТво. Рассмотрим произвольньй непрерьвный операторпродолжения

$$
G: H_{m}^{r}(\Omega) \rightarrow H_{m}^{r}\left(\mathbb{R}^{d}\right),
$$

который существует вследствие гладкости границы $\partial \Omega$ (см., например, [27]).

Оператор $R_{s}$ будем искать в следуюшем виде:

$$
R_{s} y_{0}(x)=G y_{0}(x)+\chi(x) \sum_{|\alpha| \leqslant n} c_{\alpha} \psi_{\alpha}
$$

где $n$ - такое же, как и в теореме $5.2, \chi(x)$-произвольная неотрицательная функция класса $C_{0}^{\infty}(B)$ с носителем ненулевой меры, $\left\{c_{\alpha}\right\}_{|\alpha| \leqslant n}$ - неизвестные коэффициенты, которые будут определены ниже, $\left\{\psi_{\alpha}\right\}_{|\alpha| \leqslant n}-$ функции Эрмита.

Обозначим $z_{0}(x)=R_{s} y_{0}(x)$. Включение $z_{0} \in M_{-}^{s}$ эквивалентно следуюшему уравнению:

$$
P_{+}^{n} z_{0}=g_{-}\left(P_{-}^{n} z_{0}\right)
$$

Базисом конечномерного подпространства $E_{+}^{n}$ являются функции Эрмита $\left\{\psi_{\alpha}\right\}_{|\alpha| \leqslant n}$, поэтому справедливо следуюшее представление:

$$
g_{-}(f)=\sum_{|\alpha| \leqslant n} \psi_{\alpha} g_{\alpha}(f)
$$

Из (52), (53) получаем уравнение

$$
\sum_{|\alpha| \leqslant n}\left(\int_{\mathbb{R}^{d}} P_{\alpha}\left(\xi^{\prime}\right) z_{0}\left(\xi^{\prime}\right) d \xi^{\prime}\right) \psi_{\alpha}(\xi)=\sum_{|\alpha| \leqslant n} g_{\alpha}\left(P_{-}^{n} z_{0}\right) \psi_{\alpha}(\xi) .
$$

В силу линейной независимости функций $\psi_{\alpha}(\xi)$ из предыдущего равенства выводим систему:

$$
\int_{\mathbb{R}^{d}} P_{\alpha}\left(\xi^{\prime}\right) z_{0}\left(\xi^{\prime}\right) d \xi^{\prime}=g_{\alpha}\left(P_{-}^{n} z_{0}\right), \quad|\alpha| \leqslant n
$$

Введем обозначения:

$$
\begin{aligned}
A & =\left\|a_{\alpha \beta}\right\|_{|\alpha| \leqslant n,|\beta| \leqslant n}, & a_{\alpha \beta} & =\int_{\mathbb{R}^{d}} \chi\left(\xi^{\prime}\right) P_{\alpha}\left(\xi^{\prime}\right) \psi_{\beta}\left(\xi^{\prime}\right) d \xi^{\prime}, \\
\vec{e} & =\left\{e_{\alpha}\right\}_{|\alpha| \leqslant n}, & e_{\alpha} & =\int_{\mathbb{R}^{d}} P_{\alpha}\left(\xi^{\prime}\right) G y_{0}\left(\xi^{\prime}\right) d \xi^{\prime}, \\
\vec{c} & =\left\{c_{\alpha}\right\}_{|\alpha| \leqslant n}, & \vec{g} & =\left\{g_{\alpha}\right\}_{|\alpha| \leqslant n}, \quad \vec{\psi}=\left\{\psi_{\alpha}\right\}_{|\alpha| \leqslant n} .
\end{aligned}
$$

В векторной форме система (54) после подстановки $z_{0}=R_{s} y_{0}(x)$, определенного в (51), записывается в следуюшем виде:

$$
\vec{e}+A \vec{c}=\vec{g}\left(G y_{0}+\chi(\vec{c}, \vec{\psi})-(\vec{\psi}, \vec{e}+A \vec{c})\right) .
$$

Размерность $е$ системы (55) определяется количеством мультииндексов $\alpha$, для которых $|\alpha| \leqslant n$ :

$$
e=C_{n+d-1}^{n}
$$


Докажем положительную определенность матрицы $A$. Напомним, что $P_{\alpha}$ определяется как $P_{\alpha}=e^{|\xi|^{2} / 4} \psi_{\alpha}(\xi)$. Для произвольного вектора $\eta=\left\{\eta_{\alpha}\right\}_{|\alpha| \leqslant n} \in \mathbb{R}^{e}$ будем иметь:

$$
(A \eta, \eta)=\sum_{|\alpha| \leqslant e,|\beta| \leqslant e} a_{\alpha \beta} \eta_{\alpha} \eta_{\beta}=\int_{\mathbb{R}^{d}}\left|\sum_{|\alpha| \leqslant e} \sqrt{\chi\left(\xi^{\prime}\right)} \cdot \sqrt{\frac{e^{\left|\xi^{\prime}\right|^{2}}}{4}} \cdot \psi_{\alpha} \eta_{\alpha}\right|^{2} d x \geqslant 0
$$

причем ввиду линейной независимости функций Эрмита равенство нулю достигается только в случае нулевого вектора $\eta$. Следовательно, определена обратная матрица $A^{-1}$.

Введем оператор $H: \mathbb{R}^{e} \rightarrow \mathbb{R}^{e}$ с помощью соотношения

$$
H(\vec{c})=A^{-1} \vec{g}\left(G y_{0}+\chi(\vec{c}, \vec{\psi})-(\vec{\psi}, \vec{e})-(\vec{\psi}, A \vec{c})\right)-A^{-1} \vec{e},
$$

сводящий нашу систему к уравнению

$$
H(\vec{c})=\vec{c}
$$

В силу теоремы о среднем с учетом непрерывности $\vec{g}^{\prime}$ в окрестности нуля и условия $\vec{g}^{\prime}(0)=0$ отображение $H$ является сжимаюшим при малых $\|\vec{e}\|_{\mathbb{R}^{d}},\left\|\vec{c}_{1}\right\|_{\mathbb{R}^{d}}$, $\left\|\vec{c}_{2}\right\|_{\mathbb{R}^{d}},\left\|G y_{0}(x)\right\|_{H_{m}^{r}\left(\mathbb{R}^{d}\right)}:$

$$
\begin{aligned}
& \left\|H\left(\vec{c}_{1}\right)-H\left(\vec{c}_{2}\right)\right\|_{\mathbb{R}^{e}} \leqslant C_{0} \sup _{\beta \in[0,1]} \| A^{-1} \vec{g}^{\prime}\left(G y_{0}-(\vec{\psi}, \vec{e})\right. \\
& \left.\quad+\chi\left(\beta \vec{c}_{1}+(1-\beta) \vec{c}_{2}, \vec{\psi}\right)-\left(\vec{\psi}, A\left(\beta \vec{c}_{1}+(1-\beta) \vec{c}_{2}\right)\right)\right) \|_{\mathbb{R}^{e}} \\
& \quad \times\left\|\vec{c}_{1}-\vec{c}_{2}\right\|_{\mathbb{R}^{e}} \leqslant C_{1}\left(y_{0}, \vec{c}_{1}, \vec{c}_{2}\right)\left\|\vec{c}_{1}-\vec{c}_{2}\right\|_{\mathbb{R}^{e}}
\end{aligned}
$$

где $C_{1}\left(y_{0}, \vec{c}_{1}, \vec{c}_{2}\right) \leqslant C_{2}\left(\left\|y_{0}\right\|_{H_{m}^{r}(\Omega)}+\left\|\vec{c}_{1}\right\|_{\mathbb{R}^{e}}+\left\|\vec{c}_{2}\right\|_{\mathbb{R}^{e}}\right)$.

Поэтому из принципа сжимаюших отображений следует разрешимость уравнения (55), а следовательно, и существование оператора $R_{s}$, продолжающего начальные функции $y_{0}(x)$ на инвариантное многообразие $M_{-}^{s}$.

Из непрерьвности оператора $G$ и непрерывной зависимости коэффициентов $\left\{c_{\alpha}\right\}_{|\alpha| \leqslant n}$ от функции $y_{0}$ следует непрерывность оператора $R_{s}$.

ДоКАЗАТЕЛЬСтво теоРемы 2.1. Зададимся произвольным $k>d / 4$. Положим

$$
s=k-\frac{1}{\gamma-1}+\frac{d}{4} .
$$

Пусть $z(t, x)$ - решение задачи Коши $(20),(21)$ с начальным условием $z_{0}(\cdot)=$ $R_{s} y_{0}(\cdot)$, лежащим на инвариантном многообразии $M_{-}^{s}$.

В силу того что весовая функция $\left(1+|\xi|^{2}\right)^{m / 2}$ при замене $(22)$ стремится к единище при $t \rightarrow \infty$, для решения $z(t, x)$ при каждом $t$ дальнейшие оценки будем проводить в пространстве $H^{r}\left(\mathbb{R}^{d}\right)$.

Обозначим через $\Gamma_{\Omega}, \gamma_{\partial \Omega}$ операторы, которые сужают функции, определенные в пространстве $\mathbb{R}^{d}$, на области $\Omega, \partial \Omega$ соответственно:

$$
\begin{aligned}
\Gamma_{\Omega}: H^{r}\left(\mathbb{R}^{d}\right) & \rightarrow H^{r}(\Omega), & \Gamma_{\Omega} f(x) & :=\left.f(x)\right|_{x \in \Omega} \\
\gamma_{\partial \Omega}: H^{r}\left(\mathbb{R}^{d}\right) & \rightarrow H^{r-1 / 2}(\partial \Omega), & \gamma_{\partial \Omega} f\left(x^{\prime}\right) & :=\left.f\left(x^{\prime}\right)\right|_{x^{\prime} \in \partial \Omega}
\end{aligned}
$$


Искомое решение $(y, u)$ задачи стабилизации $(15)-(17),(19)$ дается следующей формулой:

$$
(y(t, \cdot), u(t, \cdot))=\left(\Gamma_{\Omega} z(t, \cdot), \gamma_{\partial \Omega} z(t, \cdot)\right) .
$$

Докажем выполнение условия стабилизации (19). Из теорем 4.1, 6.1 с учетом $(22),(49),(50)$ и свойства непрерывности операторов $\Gamma, \gamma \partial \Omega$ мы имеем оценки:

$$
\begin{aligned}
& \left(\int_{\Omega}\left|\partial_{x}^{\alpha} y(t, x)\right|^{2} d x\right)^{1 / 2} \leqslant\left(\int_{\mathbb{R}^{d}}\left|\partial_{x}^{\alpha} z(t, x)\right|^{2} d x\right)^{1 / 2} \\
& \leqslant \frac{1}{(t+1)^{\frac{1}{\gamma-1}}}\left(\int_{\mathbb{R}^{d}}\left|\partial_{x}^{\alpha} \widehat{z}\left(\ln (t+1), \frac{x}{\sqrt{t+1}}\right)\right|^{2} d x\right)^{1 / 2} \\
& \leqslant \frac{1}{(t+1)^{\frac{1}{\gamma-1}-\frac{d}{4}+\frac{|\alpha|}{2}}}\left(\int_{\mathbb{R}^{d}}\left|\partial_{\xi}^{\alpha} \widehat{z}(\ln (t+1), \xi)\right|^{2} d \xi\right)^{1 / 2} \\
& \leqslant \frac{1}{(t+1)^{\frac{1}{\gamma-1}-\frac{d}{4}+\frac{|\alpha|}{2}}}\|\widehat{z}(\ln (t+1), \cdot)\|_{H_{m}^{r}\left(\mathbb{R}^{d}\right)}
\end{aligned}
$$

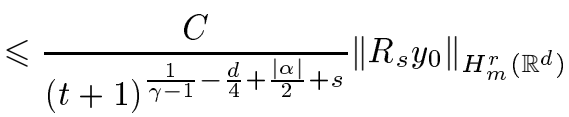

$$
\begin{aligned}
& \leqslant \frac{C}{(t+1)^{\frac{1}{\gamma-1}-\frac{d}{4}+s}}\left\|R_{s} y_{0}\right\|_{H_{m}^{r}\left(\mathbb{R}^{d}\right)} \leqslant \frac{C^{\prime}}{(t+1)^{k}}\left\|y_{0}\right\|_{H_{m}^{r}(\Omega)} \text {, }
\end{aligned}
$$

откуда следует искомая оценка для решения $y(t, x)$ :

$$
\|y(t, \cdot)\|_{H^{r}(\Omega)} \leqslant \frac{C}{(t+1)^{k}}\left\|y_{0}(\cdot)\right\|_{H_{m}^{r}(\Omega)} .
$$

И, окончательно, для управления $u$ имеем следующую оценку:

$$
\|u(t, \cdot)\|_{H^{r-1 / 2}(\partial \Omega)} \leqslant D\|z(t, \cdot)\|_{H^{r}\left(\mathbb{R}^{d}\right)} \leqslant \frac{\widetilde{D}}{(t+1)^{k}}\left\|y_{0}(\cdot)\right\|_{H_{m}^{r}(\Omega)} .
$$

\section{Список литературы}

1. Бутковский $A$. Г. Теория оптимального управления системами с распределенньми параметрами. М.: Наука, 1965.

2. Егоров Ю. В. Некоторыезадачи теории оптимального управления // ЖВМ и МФ. 1963. T. $3(5)$. C. $887-904$.

3. Егоров Ю. В. О некоторых задачах теории оптимального управления // Докл. АН ССР. 1962. T. $145(2)$. C. 241-244.

4. Гальчук Л.И. О некоторых задачах на оптимальное управление системами, описьваемыми параболическими уравнениями // Вестн. МГУ. Сер. 1. Матем., мех. 1965. Т. 1(3). C. 21-33.

5. Russel D. L. A unified boundary controllability theory for hyperbolic and parabolic partial differential equations // Stud. Appl. Math. 1973. V. 52. № 3. P. 189-211.

6. Fattoriny H. O., Russel D. L. Exact controllability theorems for linear parabolic equations in one space dimension // Arch. Ration. Mech. Anal. 1971. V. 43(3). P. 272-292. 
7. Lasiecka I., Triggiani $R$. Controllability of semilinear abstract systems with application to waves and plates boundary control problems // Appl. Math. Optim. 1991. V. 23. P. 109-154.

8. Lasiecka I., Triggiani $R$. Carleman estimates and exact boundary controllability for a system of coupled, nonconservative second-order hyperbolic equations // Partial differential equation methods in control and shape analysis / ed. G. Da Prato, J.-L. Zolésio. New York: Marcel Dekker, 1997. P. 215-243. (Lecture Notes in Pure and Appl. Math. V. 188.)

9. Triggiani $R$. Controllability and observability in Banach space with bounded operators // SIAM J. Control. Optim. 1975. V. 13 (2). P. 462-491.

10. Fursikov A. V. Exact boundary zero controllability of three-dimensional Navier-Stokes equations // J. Dynam. Control Systems. 1995. V. 1(3). P. 325-350.

11. Fursikov A. V., Imanuilov $O$. Yu. On controllability of certain systems simulating a fluid flow // Flow control. New-York: Springer-Verlag, 1995. P. 149-184. (IMA Volume Math. Appl. V. 68.)

12. Фурсиков A. В., Эмануилов О. Ю. Точная управляемость уравнений Навье-Стокса и Буссинеска // УМН. 1999. Т. 54. №3(227). С. 93-146.

13. Lasiecka I. Stabilization of hyperbolic and parabolic systems with nonlinearly pertubed boundary conditions // J. Differential Equations. 1988. V. 75(1). P. 53-87.

14. Фурсиков А. В. Стабилизируемость квазилинейного параболического уравнения с помощью граничного управления с обратной связью // Матем. сб. 2001. Т. 192(4). C. 115-160.

15. Fursikov A. V. Real process corresponding to the 3D Navier-Stokes system, and its feedback stabilization from the boundary // Amer. Math. Soc. 2002. V. 206. P. 95-123.

16. Russel D. L. Controllability and stabilizability theory for linear partial differential equations. Recent progress and open questions // SIAM Rev. 1978. V. 20. P. 639-739.

17. Seregin G., Šverák V. The Navier-Stokes equations and backward uniqueness // Preprint. IMA, 2001.

18. Гориков A.B. Степенная стабилизация одномерного уравнения теплопроводности // УМH. 2001. T. 56(2). C. 213-214.

19. Фурсиков $A$. В. Реальные процессы и реализуемость метода стабилизации системы Навье-Стокса посредством управления с обратной связью с границы области // Международная математическая серия. Нелинейные задачи математической физики и смежные вопросы. В честь академика О. А. Ладыженской. 2002. Т. 2. С. 127-164.

20. Wayne C.E. Invariant manifolds for parabolic differential equations on unbounded domains // Arch. Ration. Mech. Anal. 1997. V. 138. P. 279-306.

21. Eckmann J.-P., Wayne C.E. Non-linear stability analysis of higher order dissipative partial differential equations // Math. Phys. Electron. J. 1998. V. 4. № 3 (electronic).

22. Gallay Th., Wayne C.E. Invariant manifolds and the long-time asymptotics of the Navier-Stokes and vorticity equations on $\mathbb{R}^{2} / /$ Arch. Ration. Mech. Anal. 2002. V. 163(3). P. 209-258.

23. Лионс ЖК.-Л., Мадженес Э. Неоднородные задачи и их приложения. М.: Мир, 1971.

24. Фурсиков $A$. B. Оптимальное управление распределенными системами. Теория и приложения. Новосибирск: Научная книга, 1999.

25. Иосида К. Функциональньй анализ. М.: Мир, 1967.

26. Бабин А. В., Вишик М.И. Аттракторы эволюционных уравнений. М.: Наука, 1989.

27. Михайлов В. П. Диффференциальные уравнения в частных производных. М.: Наука, 1983.

Московский государственный

Поступила в редакцию

университет им. М.В. Ломоносова

25.02 .2003

E-mail: gorshkov@online.ru 